\title{
Interplay between wall slip and cavitation: A complementary variable approach
}

\author{
Luca Biancofiore $^{\mathrm{a}}$, Matteo Giacopini ${ }^{\mathrm{b}}$, Daniele Dini ${ }^{\mathrm{c}, *}$ \\ ${ }^{a}$ Department of Mechanical Engineering Bilkent University, 06800, Bilkent, Ankara, Turkey \\ ${ }^{\mathrm{b}}$ DIEF - Engineering Department Enzo Ferrari, via Vivarelli 10, 41125, Modena, Italy \\ ${ }^{\mathrm{c}}$ Department of Mechanical Engineering, Imperial College London, Exhibition Road, London, SW7 2AZ, United Kingdom
}

\section{A R T I C L E I N F O}

\section{Keywords:}

Cavitation

Slip condition

Complementary formulation

Surface texture

\begin{abstract}
A B S T R A C T
In this work a stable and reliable numerical model based on complementary variables is developed to study lubricated contacts characterised by slip at one or both surfaces and in the presence of cavitation. This model can be used to predict surface behaviour when cavitation induced by e.g. the presence of surface texture, slip, or a combination of the two is encountered, with varying surface parameters. For this purpose, two different algorithms are coupled to predict the formation of cavitation, through a mass-conserving formulation, and the presence of slip at the wall. The possible slippage is described by a limiting shear criterion formulated using a Tresca model. To show the flexibility of our model, several bearing geometries have been analysed, such as a twin parabolic slider, a cosine profile used to mimic a bearing, and a pocketed slider bearing employed to study the effect of surface texture. We observe that the lubrication performance (i.e. low friction coefficient) can be improved by using materials that promote slippage at the moving wall. The location of the slippage region can be optimised to find the lowest value of friction coefficient. Our theoretical developments and numerical implementation are shown to produce useful guidelines to improve and optimise the design of textured superoleophobic surfaces in the presence of lubricated contacts.
\end{abstract}

\section{Introduction}

Around $23 \%$ of the worlds total energy consumption originates from tribological contacts, most of it used to overcome friction [1]. For this reason, recently, interest in the field of friction reduction has grown significantly [2-4]. More and more efficiency savings must be made in mechanical systems, since stricter emissions standards are enforced. Particularly in the long term the implementation of advanced tribological technologies for friction reduction and wear protection, could bring in the long term to (i) saving about $8.7 \%$ of the total energy consumption (1.4\% of the world GDP) and (ii) reduce the $\mathrm{CO}_{2}$ emissions globally by as much as $1460 \mathrm{MtCO} 2$ (i.e. 450,000 million Euros cost savings) [1]. At the same time, the miniaturisation of devices, particularly MEMS (microelectricalmechanical systems) poses an additional challenge. Friction is a limiting factor in the development of such devices [5]. Through the application of new friction reducing technologies, less powerful and smaller motors and pumps can be delivered, meaning that current trends in miniaturisation can be continued. Experimental and theoretical studies have shown that, if properly optimised, both surface texture and artificially induced slip can individually reduce the friction of a system. Results have proved that surface texture can reduce friction by up to $75 \%$ in some conditions [6], and slip by more than $40 \%$, depending on surface parameters [7].

Surface texturing has become a viable engineering technique to improve the tribological performance of surfaces, capable of significantly improving load capacity, wear resistance, and friction reduction. It involves producing a surface with micro-dimples, or other texture shape, through various means of manufacture which are used in applications where lubricated surfaces are sliding relative to each other, traditionally seals and bearings. When moving relative to another body, the dimples can act either as a microhydrodynamic bearing (full and mixed lubrication), or a micro-reservoir for lubricant (mixed and starved) [8]. Texture can also act as a debris trap, hence contributing to reduce wear [9]. A fundamental aspect to consider in surface textured contacts is cavitation, which is usually referred to describe a complex thermodynamical process including nucleation, growth and implosion of vapour or gas bubbles within a liquid. Cavitation can be beneficial for fluid lubrication by promoting the lubricant entrainment and consequently reducing friction [10]. Furthermore the transient interaction between cavitating fluid due to textured surfaces adopted in

\footnotetext{
* Corresponding author.

E-mail address: d.dini@imperial.ac.uk (D. Dini).
} 
reciprocating surfaces has also been shown to have potential benefits, and improved design of piston-rings contacts have been proposed based on this evidence $[11,12]$.

The universally accepted no-slip condition is nothing more than an approximation that holds experimentally only under normal flow conditions [13]. Recent works have shown that surface engineering, particularly superhydrophobic surfaces, can be used to modify the no-slip condition, i.e. induce slip at a solid-fluid interface. With slip conditions at the boundaries a lower pressure is needed to move the same amount of fluid, requiring less energy. There are two main wall slip models which have been adopted to describe the slip boundary: (i) the slip length model [14] and (ii) the critical shear stress model or Tresca model $[15,16]$. Extensive theoretical work by Spikes [16] based on the Tresca model has concluded that a superhydrophobic surface is suitable for use in bearings due to its ability to not be fully wetted by a fluid. His analyses on bearings with different properties showed that for surfaces where the critical shear stress was set to be approaching zero, very low friction was recorded when compared with conventional bearings. Afterwards Li and co-workers coupled other effects such as the (i) flow rheology [17] and (ii) surface roughness [18] to anisotropic boundary slip to more accurately evaluate the performance of journal bearings. Ma and co-workers $[19,20]$ have proposed an extension of the Tresca slip-model for the analysis of two-dimensional journal bearings. They assumed a polygonal approximation of the theoretical slip-limit circle and identified suitable complementarity variables to solve the slip problem adopting quadratic programming techniques. They found that an optimum bearing design exists with a slip zone in the inlet region, capable to provide high load supports and low friction coefficients. Finally molecular dynamics is gaining ground in studies about the wall slip phenomenon [21].

But is it possible to combine the effect of wall slip and surface textures? Starting from the pioneering work of Rao [22] several papers on the subject were published, see e.g. the review by Senatore \& Rao [23]. Aurelian et al. [24] investigated the influence of wall slip over the load support and power loss in hydrodynamic fluid bearings using a simple textured/wall slip combination pattern. The main conclusion of their study was that choosing the texture/slip zone geometry should be done carefully because inappropriate choice can lead to a drastic deterioration of the bearing performance. Tauviqirrahman and co-workers [25-29] explored the possibility of slip and texture in order to improve the performance characteristics of simple bearings using both numerical and analytical models. Their results indicate that the combined texture/slip pattern having low texture cell aspect ratio, low critical shear stress, and high slip length configuration can increase the load carrying capacity and decrease friction. Lin et al. [30] performed a numerical analysis on the effects of combined surface texture and slip while trying to optimise the surface design in bearing applications. In particular they investigated the effect of adding a texture/slip surface to only parts of a journal bearing.

While all these papers have showed that both surface texture and slip can theoretically cause a significant reduction in the friction coefficient, there is only limited experimental evidence of this behaviour in the literature. However, before conducting expensive and time-demanding experiments a careful optimisation of the surface parameters is needed. Particularly it is necessary to understand how - and if - it is possible to maximise the bearing performance while minimising the increase in manufacturing cost. Our approach is similar to a recent work conducted by Bayada [31] but using a different model for cavitation and a different numerical technique. The aim of the present paper is to produce robust numerical schemes to enable researchers to explore a wide parameter space in for the optimisation of patterned surfaces in the presence of micro-textures and oleo-/hydro-phobic coatings and therefore to fill this gap. We develop an innovative and accurate numerical model to be able to predict simultaneously the slip at the wall, by using the Tresca model, and the formation of cavitation. The developed algorithm is based on the concept of complementarity.
This technique was first applied to deal with unilateral contacts in structural mechanics [32] and then applied to individually describe slip at the wall $[33,34]$ and cavitation $[35,36]$. The flexibility of the complementary algorithm is fundamental to predict the behaviour of contact with extreme thickness gradients, typical of textured surfaces (such as dimple or pockets, for instance). A preliminary one-dimensional version of the algorithm is employed here in order to speed-up the analyses necessary for the testing of the solver and to prove the validity of the newly proposed framework. Therefore, only suitable test cases considering infinitely long bearings are selected for comparison to existing solutions of similar problems available in the literature. The algorithm can be extended for the analysis of two-dimensional problems (finite length bearings) following e.g. the methodologies presented in Ref. [20] for the slip problem and in Ref. [36] for the mass-conserving cavitation problem.

The paper is structured as follows: in section 2 we introduce the basic equations, while the complementary formulations for predicting both the cavitation and slip at the wall are described in section 3. The numerical methods are described in section 4 just before showing the results for different contact profiles presented in section 5. Finally conclusions are drawn in section 6 .

\section{Basic equations}

Consider the Reynolds equation for the compressible, quasistationary, unidimensional, isothermal, isoviscous, hydrodynamic problem in which cavitation is described via the mass-conserving model introduced by Giacopini et al. [35].

$\frac{d}{d x}\left(\frac{f(p) h^{3}}{6 \mu} \frac{d p}{d x}\right)-\left[\frac{d(f(p) h U)}{d x}-\frac{d(r h U)}{d x}\right]=0$,

where $r=1-\frac{\rho}{f(p) \rho_{0}}, \rho$ is density of fluid, $\rho_{0}$ is the density of the fluid at the inlet $x=0$, and $f(p)$ is the functional connection between $\rho$ and $p$ [36]. In the active region (full film) the density is equal to $f(p) \rho_{0}$, while in the non-active region (cavitated area) the density can vary and in general is lower or equal to $\rho_{0}$. With the assumption of no-slip conditions at both upper and lower profiles, $U$ is usually employed to define both the velocity of the lower sliding wall and the velocity of the fluid layer in contact with it. At the same time, a null velocity is considered for the fluid layer in contact with the upper fixed surface. In addition, $p$ is the fluid gauge pressure, $h$ is the film thickness, and $\mu$ is the fluid viscosity. Note that the saturation pressure in our model is assumed to be zero without losing in generality since $p$ is defined as the gauge pressure.

Equation (1) can then be modified considering the possibility of fluid slippage. In particular, the present contribution assumes that a slippage can occur only between the fluid and the upper fixed surface, namely the one identified by $z=h$, see the representative schematic in Fig. 1. As a consequence, a second velocity $U_{S}$ is introduced in order to describe the possible relative motion between the fluid and the upper

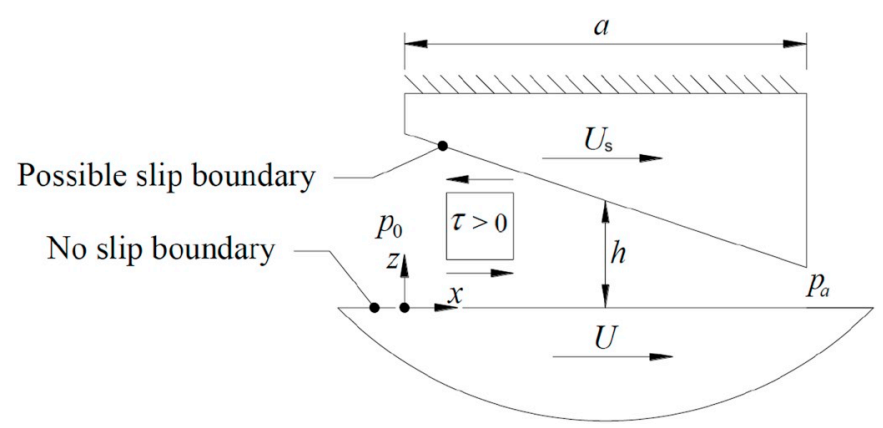

Fig. 1. The model analysed for the tangential velocity slip problem. 
fixed surface. Therefore equation (1) becomes:

$\frac{d}{d x}\left(\frac{f(p) h^{3}}{6 \mu} \frac{d p}{d x}\right)-\left[\frac{d\left(f(p) h\left(U+U_{s}\right)\right)}{d x}-\frac{d\left(r h\left(U+U_{s}\right)\right)}{d x}\right]=0$.

Suitable boundary conditions can then be chosen together with Equation (2):

$p(0)=p_{0} \quad$ and $\quad p(a)=p_{a}$

where $a$ is the extent of the lubricated region.

In order to clarify the geometry of the problem and the meaning of the main involved parameters, the schematic of the standard hydrodynamic converging bearing is depicted in Fig. 1. In the following, the fluid shear stress at the interface between the fluid layer and the upper fixed surface, $\tau$, is expressed as a function of the velocity of the same fluid layer, $U_{s}$. First of all, we integrate twice Equation (2). Taking into account the boundary conditions of Equation (3), the expression for the fluid pressure, $p$, is then derived as a function of $U_{s}$

$$
\begin{aligned}
p(x)=6 \mu \int_{0}^{x}(U+ & \left.U_{S}\right) \frac{f(p)-r}{f(p) h^{2}} d x^{\prime}-6 \mu \frac{\int_{0}^{a}\left(U+U_{s}\right) \frac{f(p)-r}{f(p) h^{2}} d x^{\prime}}{\int_{0}^{a} \frac{1}{f(p) h^{3}} d x^{\prime}} \int_{0}^{x} \frac{1}{f(p) h^{3}} d x^{\prime}+ \\
& +\left(p_{a}-p_{0}\right) \frac{\int_{0}^{x} \frac{1}{f(p) h^{3}} d x^{\prime}}{\int_{0}^{a} \frac{1}{f(p) h^{3}} d x^{\prime}}+p_{0} .
\end{aligned}
$$

Then, the shear stress at the interface between the fluid and the upper fixed profile, i.e. at $z=h$, can be written as

$\left.\tau\right|_{z=h}=-\frac{h}{2} \frac{d p}{d x}+\left(U-U_{s}\right) \frac{\mu}{h}$

The reader has to refer to Fig. 1 for the sign convention and see the derivation of Equation (B.5) in Appendix B for more details. The sign of the shear stress in Equation (5) agrees with the physical reasoning introduced in Ref. [15]. In fact, when the fluid slips at the boundary with a solid surface, the shear stress at the interface always opposes the slip. Therefore, it is natural to consider it as positive if the relative velocity between the fluid and the wall is negative and vice versa.

Finally, substituting the derivative of Equation (4) into Equation (5), the shear stress at the interface between the fluid layer and the upper fixed surface, $\tau$ can be derived as a function of the velocity of the same fluid layer, $U_{s}$

$$
\begin{gathered}
\left.\tau\right|_{z=h}=-\frac{\mu(4 f(p)-3 r) U_{S}}{f(p) h}+\frac{3 \mu \int_{0}^{a} \frac{U_{S}(f(p)-r)}{f(p) h^{2}} d x^{\prime}}{f(p) h^{2} \int_{0}^{a} \frac{1}{f(p) h^{3}} d x^{\prime}}+\frac{3 \mu \int_{0}^{a} \frac{U(f(p)-r)}{f(p) h^{2}} d x^{\prime}}{f(p) h^{2} \int_{0}^{a} \frac{1}{f(p) h^{3}} d x^{\prime}}+ \\
+\frac{\mu U(3 r-2 f(p))}{f(p) h}-\frac{p_{a}-p_{0}}{2 f(p) h^{2} \int_{0}^{a} \frac{1}{f(p) h^{3}} d x^{\prime}} .
\end{gathered}
$$

\section{Formulation in terms of complementarity}

In this section we formulate the two intertwined problems (i.e. cavitation and slippage at the upper wall) in terms of complementarity. In particular in section 3.1 the complementarity formulation to describe cavitation is presented while in section 3.2 the formulation to model the possible slippage is introduced.

\subsection{Cavitation}

As already observed in the previous section the pressure is larger than zero in the active region while it is assumed to be equal to zero in the non-active region. At the same time the parameter $r$ is null in the active region while it is strictly positive in the cavitated region.

For this reason we can define the complementary formulation related to the problem of cavitation in lubricant films in presence of slip as: $\frac{d}{d x}\left(\frac{f(p) h^{3}}{6 \mu} \frac{d p}{d x}\right)-\left[\frac{d\left(f(p) h\left(U+U_{S}\right)\right)}{d x}-\frac{d\left(r h\left(U+U_{S}\right)\right)}{d x}\right]=0$,

$p \geq 0$,

$r \geq 0$ and

$p r=0$.

Equation (7a) can be rewritten in a compact functional form

$p=L_{c} r+Q_{c}$,

where $L_{c}$ and $Q_{c}$ can be easily found comparing eqs. (4) and (8). The reader is referred to Refs. [35,36] for more details on the complementarity formulation regarding cavitation.

\subsection{Slippage}

This section presents the formulation of the tangential velocity slip problem in terms of complementary variables. In particular, the Tresca model is employed in order to mimic the slippage between the fluid and the solid surfaces. In this paper we focus on the case of the possible slippage just in the positive streamwise direction as in the preliminary work of Strozzi [33]. However, an algorithm based on variational inequalities which naturally incorporates the possibility of slippage in the two opposite directions can be developed since it is relatively easy to consider a finite interval for the values assumed by the shear stress at the slippage surface, $\tau$, see Ref. [34].

In detail, the physical significance of the problem under examination requires that, if the shear stress $\tau$ is lower than the critical value $\tau_{c}$ no slippage occurs, and therefore $U_{S}=0$. If $\tau$ reaches its critical upper bound $\tau_{c}$, the fluid velocity can be higher than that of the upper boundary, $U_{s} \geq 0$. Thus we can arrive to the complementary formulation

$\left(U_{s}, \tau_{c}-\tau\right) \geq 0$,

in which $\tau \leq \tau_{c}$. More details can be found in Ref. [37], to which the interested reader is referred.

First of all we can write eq. (6) again in a compact form

$\tau=L_{s} U_{s}+Q_{s}$,

where $L_{s}$ and $Q_{s}$ can be easily identified by comparing eqs. (6) and (10). Once the complementary formulation governing the problem is defined, the functional connection between the two complementary variables of eq. (9) can be easily identified manipulating eq. (10):

$\tau=L_{s} U_{s}+Q_{s} \Rightarrow-U_{s}=-L_{s}^{-1} \tau+L_{s}^{-1} Q_{s} \Rightarrow-U_{s}=M \tau+P$,

where operator $M$ of equation (11) equals the inverse of $L_{s}$ appearing in equation (10) changed in sign. In conclusion, the tangential velocity slip problem can be expressed in the following way: find $\tau \leq \tau_{c}$ such that.
1. $-U_{s}=M \tau+P$
2. $\left(U_{s}, \tau_{c}-\tau\right) \geq 0 \quad \tau \leq \tau_{c}$.

In this paper we take into account the possibility that the critical value depends on the streamwise direction $\tau_{c}(x)$. The whole slip problem can be then stated via a typical complementarity approach as follows:

$M^{-1} U_{s}+M^{-1} P=\tau_{c}(x)-\tau$,

$\left(\tau_{c}(x)-\tau\right) \geq 0$,

$U_{s} \geq 0$ and

$U_{S}\left(\tau_{c}(x)-\tau\right)=0$.

The extension to negative threshold for the shear stress is straightforward and the reader is referred to Ref. [34] for further details. 


\section{Numerical method}

The algebraic description for both complementary problems presented in the previous sections can then be implemented with few efforts in the Finite Element framework. In the following a brief description of the numerical method employed is presented. The reader is referred to Refs. [34-36] to have more details about these numerical methods.

\subsection{Cavitation problem}

We start to rewrite eq. (7a) applying the standard Galerkin method

$$
\begin{aligned}
F & =\int_{\Omega} W\left(\frac{\partial}{\partial x}\left(\frac{f(p) h^{3}}{6 \mu} \frac{\partial p}{\partial x}\right)-\frac{\partial\left(f(p) h\left(U+U_{s}\right)\right)}{\partial x}+\frac{\partial\left(r h\left(U+U_{s}\right)\right)}{\partial x}\right) d \Omega \\
& =0,
\end{aligned}
$$

where $W$ is a basis function used in the entire solution domain $\Omega$. Considering suitable shape functions $N$, the following system of linear algebraic equations can be obtained

$F=A p+B r+C=0$,

where

$A_{j, k}=-\frac{1}{6 \mu} \sum_{n=1}^{N_{e}} \sum_{m=1}^{N_{g p}}\left(f\left(p_{i-1}\right)_{m} h_{m}^{3} \sum_{k=1}^{N_{n}} \frac{\partial N_{m k}}{\partial x} \frac{\partial W_{m j}}{\partial x}\right) w_{m} \Delta \Omega_{m}$,

$B_{j, k}=\sum_{n=1}^{N_{e}} \sum_{m=1}^{N_{g p}}\left(\sum_{k=1}^{N_{n}} W_{m j} h_{k}\left(U+\left(U_{s, I-1}\right)_{k}\right) \frac{\partial N_{m k}}{\partial x}\right) w_{m} \Delta \Omega_{m} \quad$ and

$C_{j}=-\sum_{n=1}^{N_{e}} \sum_{m=1}^{N_{g p}}\left(W_{m j} \frac{\partial\left(f\left(p_{i-1}\right)_{m} h_{m}\left(U+\left(U_{s, I-1}\right)_{m}\right)\right)}{\partial x}\right) w_{m} \Delta \Omega_{m}$.

Note that the $m$-index defines a general Gauss point, $w_{m}$ is the corresponding weight, $\Delta \Omega_{m}$ is the determinant of the coordinate transformation from the global coordinate system to the local element coordinate system, the $k$-index defines a general element node and $N_{g p}$, $N_{e}$ and $N_{n}$ represent the number of Gauss points per elements, the number of elements and the nodes for each element, respectively.

It should be noted that there is a loop in place in the cavitation problem (whose iterations are called $i$ ). This loop is necessary since the compressibility function $f(p)$ has to be evaluated explicitly using the pressure values at the previous iteration $i-1$. A relaxation factor $\alpha_{c}$ was also introduced to ensure convergence

$p_{i}=\alpha_{c} p_{\text {calc }}+\left(1-\alpha_{c}\right) p_{i-1}$,

in which $p_{\text {calc }}$ is the pressure obtained solving complementarity eq. (8) for the $i$-th iteration. After some tests, $\alpha_{c}=0.8$ was found to be the most suitable value. For all the analyses presented, a relative convergence tolerance equal $10^{-9} \%$ has been considered for $f(p)$.

From eq. (14) we can find the pressure by inverting matrix $A$

$p=-A^{-1} B r-A^{-1} C$

and by comparing with eq. 8

$L_{c}=-A^{-1} B$ and $Q_{c}=-A^{-1} C$.

If we consider the complementary conditions in eqs. (7b) and (7d) the system of eq. (17) can be solved via a pivoting algorithm for complementary problems [32].

\subsection{Slippage problem}

The matrices $L_{s}$ and vector $Q_{s}$, which govern the slippage problem (eq. (10)), can be easily derived comparing eq. (6) and eq. (10). Numerical implementation of the slippage problem is confined to the one-

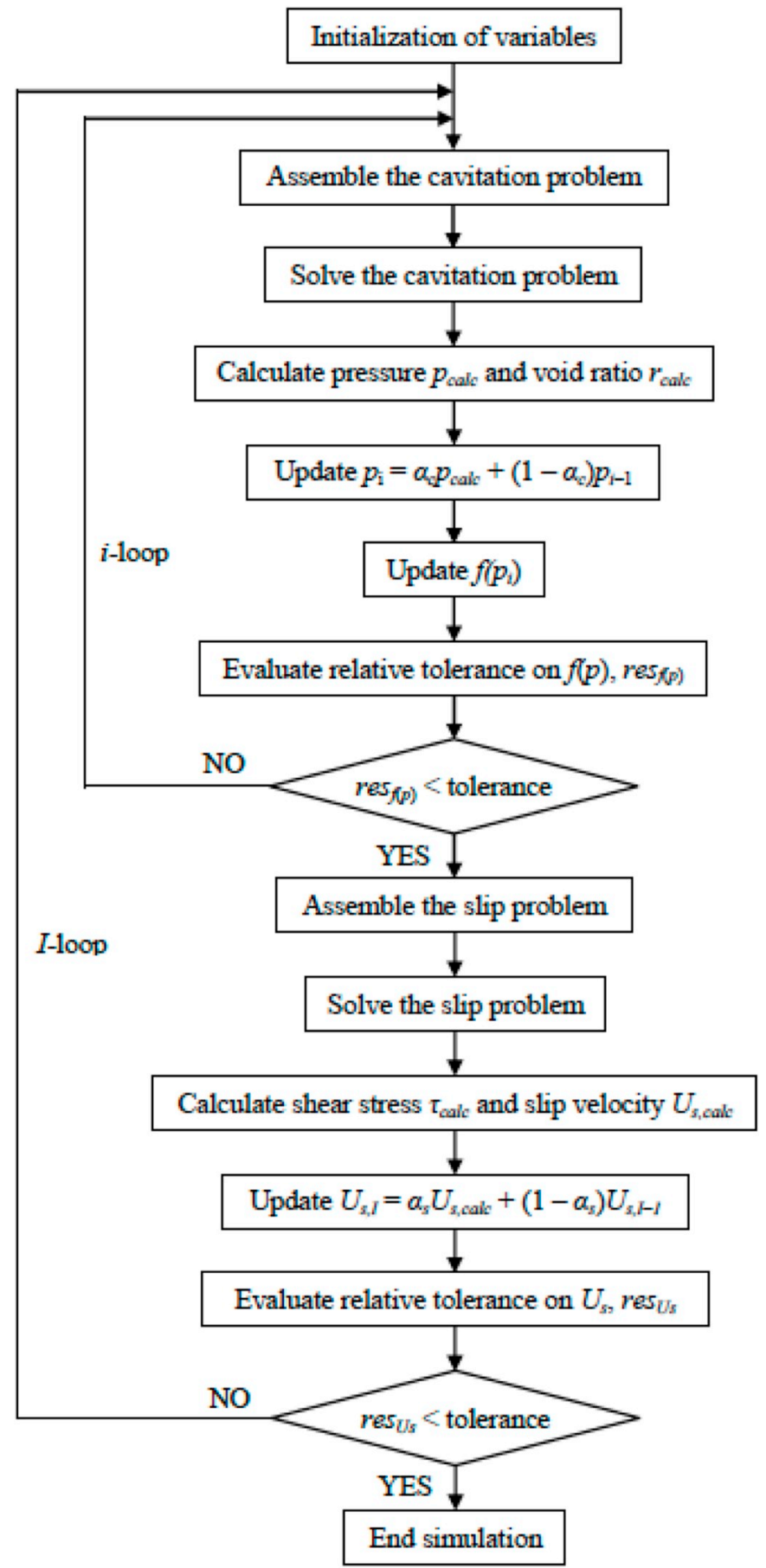

Fig. 2. The flow chart of the algorithm used in the present paper.

dimensional case and it is therefore quite simple. In particular, it can be noted that the only term which requires the numerical evaluation of an integral involving one of the complementarity variable $\left(U_{s}\right)$ is the second term of eq. (6). All the other terms return simple scalars evaluated at the $\mathrm{j}$-th node of the discretised domain. It is possible to write then

$L_{s, j, k}=-\frac{\mu\left(4 f\left(p_{i}\right)_{j}-3 r_{j}\right)}{f\left(p_{i}\right)_{j} h_{j}}+$

$\frac{3 \mu}{f\left(p_{i}\right) h_{j}^{2} \mathscr{J}_{3}} \sum_{n=1} N_{e} \sum_{k=1} N_{n} \sum_{m=1} N_{g}\left(N_{m, k} \frac{f\left(p_{i}\right)_{m}-r_{m}}{f\left(p_{i}\right) m h_{m}^{2}}\right) w_{m} \Delta \Omega_{m}$,

$Q_{s, j}=\frac{3 \mu U \mathscr{I}_{2}}{f\left(p_{i}\right)_{j} h_{j}^{2} \mathscr{I}_{3}}+\frac{\mu U\left(3 r_{j}-2 f\left(p_{i}\right)_{j}\right)}{f\left(p_{i}\right)_{j} h_{j}^{2}}-\frac{p_{a}-p_{0}}{2 f\left(p_{i}\right)_{j} h_{j}^{2} \mathscr{I}_{3}}$, 


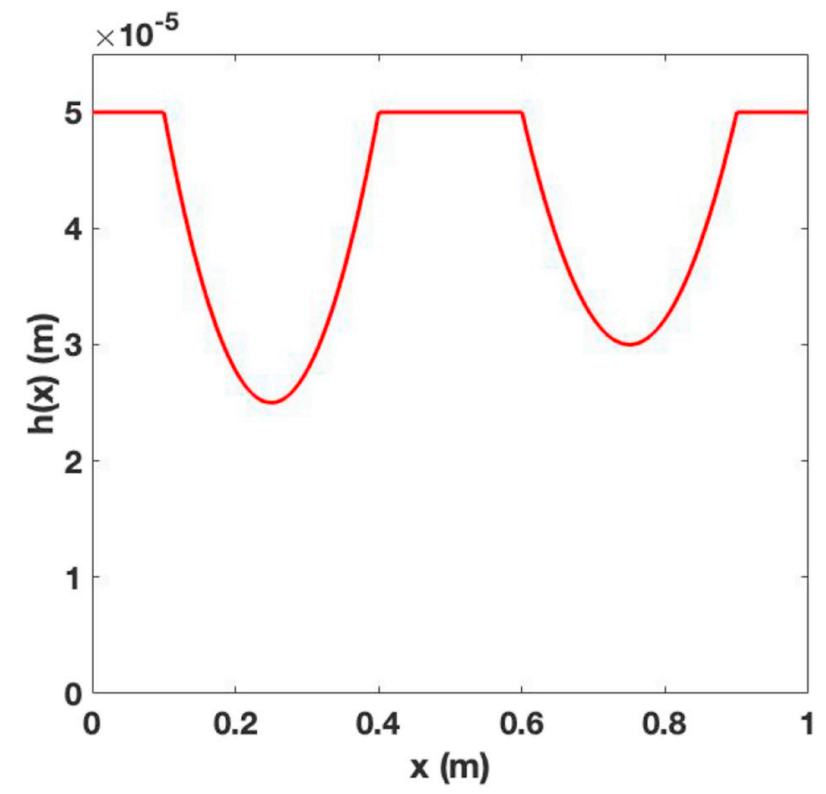

Fig. 3. The twin parabolic slider profile used in Ref. [31].

where

$\mathscr{I}_{2}=\sum_{n=1} N_{e} \sum_{m=1} N_{g}\left(\sum_{k=1} N_{n}\left(N_{m, k} \frac{1}{f\left(p_{i}\right) k h_{k}^{3}}\right)\right) w_{m} \Delta \Omega_{m} \quad$ and

$\mathscr{I}_{3}=\sum_{n=1} N_{e} \sum_{m=1} N_{g}\left(\sum_{k=1} N_{n}\left(N_{m, k} \frac{f\left(p_{i}\right)_{k}-r_{k}}{f\left(p_{i}\right) h_{k}^{2}}\right)\right) w_{m} \Delta \Omega_{m}$

\subsection{Link between the two formulations}

A second loop whose iterations are named $I$ is necessary to link the cavitation problem with the evaluation of wall slip. The values of $U_{s}$ in eq. (15) are not known a priori and need to be obtained by solving eq. (12). For this reason the slip velocity $U_{s}$ at the previous iteration $I-1$ is used in eq. (15).

Another relaxation factor $\alpha_{s}$ is required to ensure convergence of this second loop

$U_{s, I}=\alpha_{s} U_{s, \text { calc }}+\left(1-\alpha_{s}\right) U_{s, I-1}$

where $U_{s, \text { calc }}$ is the slip velocity calculated from the complementary problem illustrated by eq. (12) at the $I$-th iteration. A value of $\alpha_{s}=0.9$

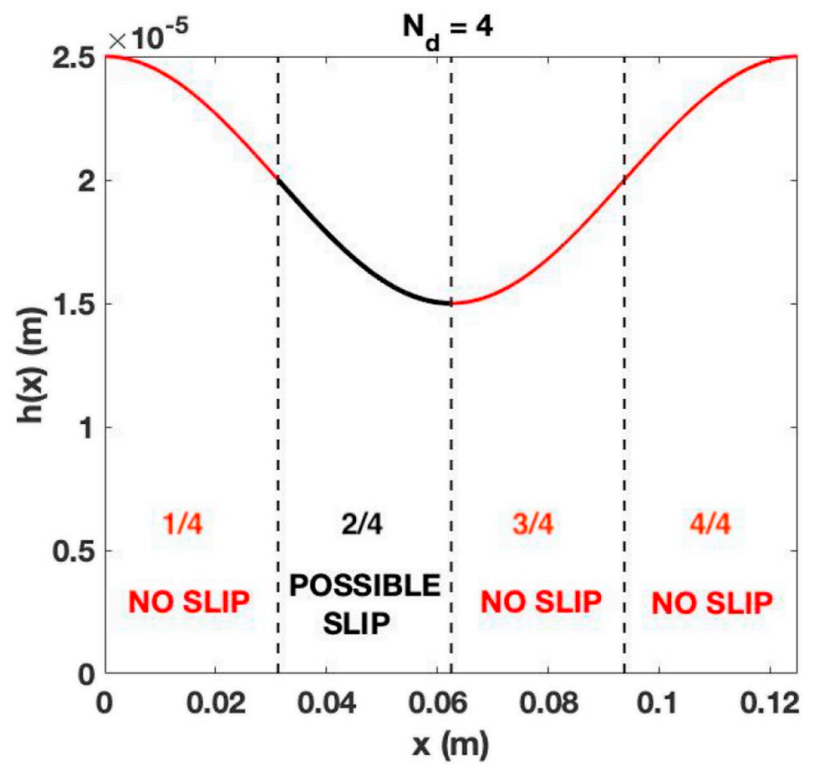

Fig. 5. The bearing modelled as a cosine profile. Note that the critical shear stress can be uniform or non-uniform. In particular, in this figure a specific nonuniform configuration (" $N_{d}=4,2 / 4$ ") is depicted.

was found through testing to be the most suitable to reach convergence. For all the analyses presented, a relative convergence tolerance equal $10^{-9} \%$ has been considered for $U_{s}$.

The algorithm used in this work is summarised in the flow chart of Fig. 2.

\section{Numerical results and discussion}

In this section we report the simulations results using different contact profiles. First of all, in section 5.1 we validate our code comparing our results with those very recently presented by Bayada [31] using an alternative approach. This is followed by the results obtained for a cosine profile used as an approximation to model a bearing in section 5.2. Finally, pocketed sliders adopted to model surface textured geometries are presented in sections 5.3 and 5.4. In particular, the multi-pocket profile of section 5.4 allows to prove the predictive capabilities of our cavitation algorithm since this kind of profile is well described just by mass conserving cavitation algorithms. In most of the simulations we have used $N_{x}=400$ as number of grid points. We have
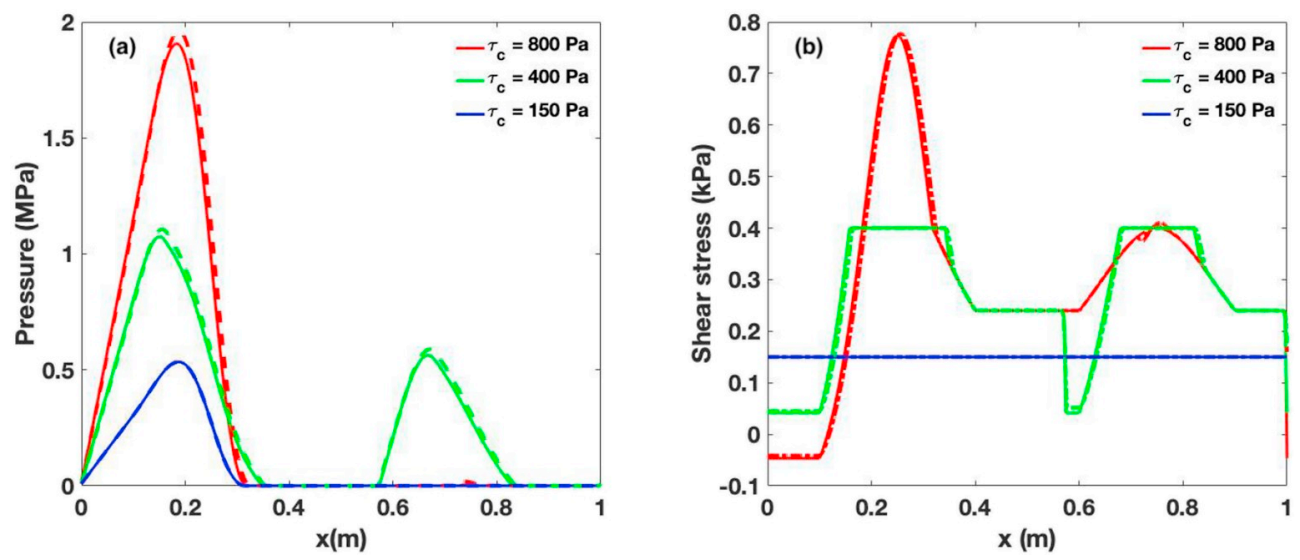

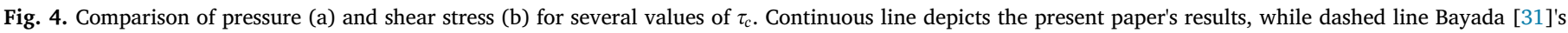
result. 

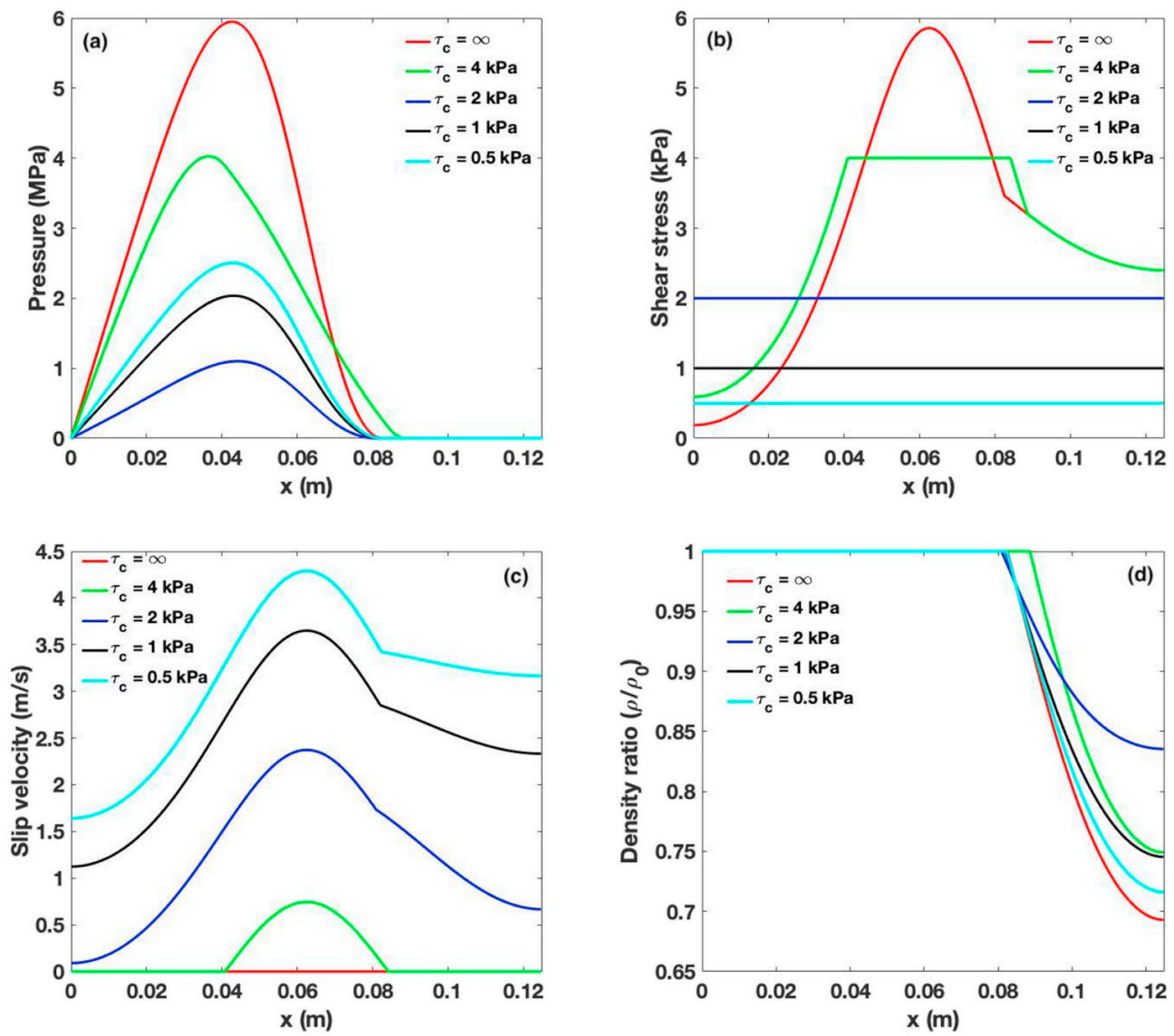

Fig. 6. Pressure (a), shear stress (b), slip velocity (c) and density ratio (d) for the cosine profile for several values of uniform $\tau_{c}$.

chosen this number after conducting grid independency tests. For the case of the twin parabolic slider only $N_{x}$ was chosen to be $N_{x}=500$ in order to use the same amount of grid points used in Ref. [31].

\subsection{Validation with a twin parabolic slider}

In this section we validate our results by comparing with a recent paper of Bayada [31]. Particularly a twin parabolic slider profile (see Fig. 3) was used in which the thickness is given by

$h(x)=\left\{\begin{array}{ccc}h_{\max }\left(A_{1} x^{2}+B_{1} x+C_{1}\right) & \text { for } & L_{1}-\Delta L_{1}<x<L_{1}+\Delta L_{1} \\ h_{\max }\left(A_{2} x^{2}+B_{2} x+C_{2}\right) & \text { for } & L_{2}-\Delta L_{2}<x<L_{2}+\Delta L_{2} \\ h_{\max } & & \text { otherwise, }\end{array}\right.$

where $\quad A_{1,2}=\frac{1-h_{1,2}}{\Delta L_{1,2}^{2}}, \quad B_{1,2}=-2 A_{1,2} L_{1,2}, \quad C_{1,2}=1-A_{1,2}$ $\left(L_{1,2}-\Delta L_{1,2}\right)^{2}+2 A_{1,2} L_{1,2}-\Delta L_{1,2}, L_{1}=0.25 a, L_{2}=0.75 a, \Delta L_{1}=\Delta L_{2}=$ $0.15 a, h_{1}=\frac{1}{2}, h_{2}=\frac{3}{5}, h_{\max }=5 \cdot 10^{-4} \mathrm{~m}$ and the length of the domain is $a=1 \mathrm{~m}$. Other parameters are $\mu=0.04 \mathrm{~Pa} \cdot \mathrm{s}, p_{a}=p_{0}=8.5 \cdot 10^{2} \mathrm{~Pa}$ and $U=0.3 \frac{\mathrm{m}}{\mathrm{s}}$.

We have used the following pressure-density relationship to model the compressibility of the liquid

$f(p)=\exp \left(\frac{p}{\beta}\right)$, in which the bulk modulus $\beta$ was set equal to $\beta=2.2 \cdot 10^{9} \mathrm{~Pa}$. This relationship was chosen since it accurately reproduces the compressibility model of Refs. [31,38] in the pressure range reached in the present work.

In Fig. 4 the pressure and the shear stress are illustrated for three different values of the critical shear stress $\tau_{c}: \tau_{c}=800 \mathrm{~Pa}$ (red line), $\tau_{c}=400 \mathrm{~Pa}$ (green) and $\tau_{c}=150 \mathrm{~Pa}$ (blue), which is assumed uniform along the contact. The continuous lines represent the values obtained through the model described in this paper while the dashed lines are extracted from Ref. [31]. The two algorithms show a very similar behaviour in which the difference is around $2 \%$ at the first peak of the pressure for $\tau_{c}=800 \mathrm{~Pa}$ and $\tau_{c}=400 \mathrm{~Pa}$. This difference is most probably due to the different cavitation models used in the two algorithms which results in predicting a slightly different starting point for the nonactive region. In particular, Bayada's algorithm treats the flow as a homogenous mixture in which the cavitation can be quantified and the pressure can fall below the cavitation pressure [38].

We are now confident in the accuracy and the predictive power of our algorithm and therefore in the next section it can be applied to different geometries. However, it should be noticed that the compressibility effect is negligible for the pressure values reached in Fig. 4a. In the following sections the pressure distributions computed for the cases under investigation will have the same order of magnitude; this implies that compressibility effect can be discarded and to simplify the analysis the density is assumed to be constant in the non-active fluid region. 

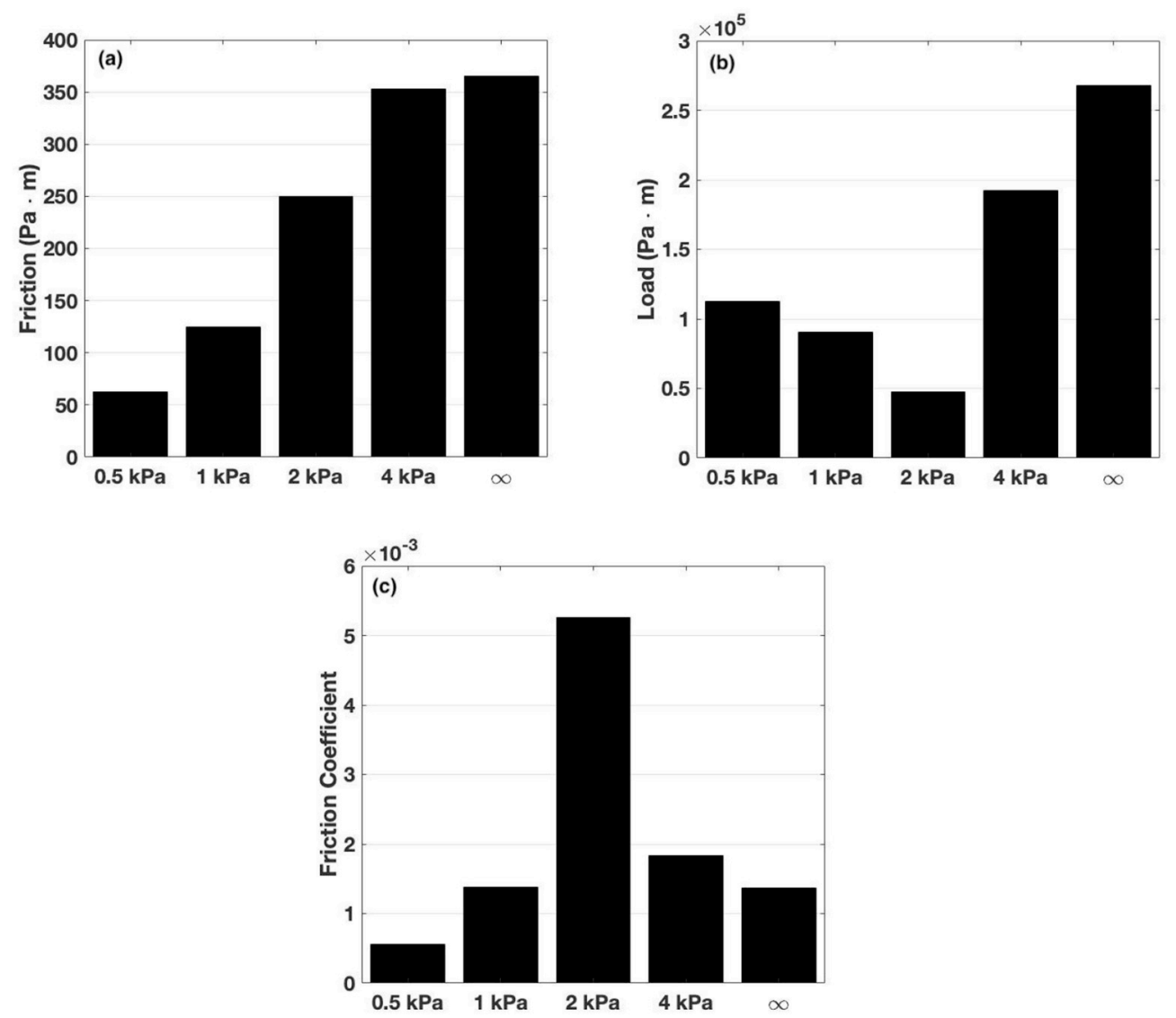

Fig. 7. Friction (a), load (b), and friction coefficient (c) for the cosine profile for several values of uniform $\tau_{c}$.

\subsection{Bearing}

In this section we model a bearing by using a cosine profile. In particular the chosen profile (shown in Fig. 5) is

$h(x)=c\left[1+\frac{\varepsilon}{c} \cos \left(\frac{2 \pi \omega x}{a}\right)\right]$,

where $c$ is the clearance $c=2 \cdot 10^{-5} \mathrm{~m}, \varepsilon$ the eccentricity $\varepsilon=0.5 \cdot 10^{-5}, \omega$ the frequency is equal to $\omega=1 \mathrm{~m}^{-1}$, and $a=0.125 \mathrm{~m}$. Other parameters are: $\mu=0.015 \mathrm{~Pa} \cdot \mathrm{s}, U=4 \mathrm{~m} / \mathrm{s}$ and $p_{a}=p_{0}=0$. This profile was already used by Giacopini et al. [35] but considering pure no-slip boundary conditions at both walls.

Results assuming a uniform critical shear stress are reported in section 5.2.1, while in section 5.2.2 non-uniform $\tau_{c}$ will be considered. Particularly in the latter case the profile is divided in $N_{d}$ equal parts and just in $1 / N_{d}$-th of the profile a finite value of the critical shear stress is considered, while in the other parts a no-slip boundary condition is implemented, i.e. $\tau_{c}=\infty$. An example is shown in Fig. 5 where the black line depicts the possible slippage region. This configuration mimics the case in which just a partial surface of the bearing is superoleophobic. A specific notation is used to identify the area where slip can occur as exemplified below. For instance if we divide the profile in $N_{d}=4$ parts and we apply the slippage in the second quarter only, we define this configuration as " $N_{d}=4,2 / 4$ ".

\subsubsection{Uniform critical shear stress}

In this section bearing profiles with a uniform critical shear stress are considered (i.e. " $N_{d}=1,1 / 1$ ”).

In Fig. 6 (a) the pressure, (b) the shear stress, (c) the slip velocity and (d) the density ratio are illustrated for different values of $\tau_{c}$. The configuration $\tau_{c}=\infty$ is equivalent to set a no slip boundary condition on the upper wall as well. For finite but large values of the critical shear stress (i.e. $\tau_{c}=4 \mathrm{kPa}$ ) the pressure diminishes; however this trend reverts and the pressure values grow again from values below $\tau_{c}=2 \mathrm{kPa}$, see Fig. 6a. The change in trend is due to the fact that entering in this range of $\tau_{c}$ the shear stress is constant and equal to the critical value. This means that the fluid is allowed to slip along the entire upper wall as one can notice by observing the shear stress or the slip velocity in Fig. $6 \mathrm{~b}$ or $6(\mathrm{c})$. The film rupture boundary (i.e. the beginning of the non-active region) does not significantly vary with reducing the critical shear stress. A reduction of the cavitated area is observed only for $\tau_{c}=4 \mathrm{kPa}$ (see Fig. $6 \mathrm{~d}$ ). This occurs since in this configuration the fluid slips just in a region and not all along the domain.

In Fig. 7 (a) the friction $F=\int_{0}^{L} \tau(x) d x$, (b) the load $W=\int_{0}^{L} p(x) d x$ and (c) the friction coefficient $F C=\frac{F}{W}$ are illustrated for the same values of $\tau_{c}$ chosen in Fig. 6. Not surprisingly friction is always decreasing by lowering the critical shear stress, while the load follows the pressure behaviour: it decreases (increases) for moderate (low) $\tau_{c}$. However, the most relevant parameter to measure the bearing performance is the friction coefficient. The performance initially 

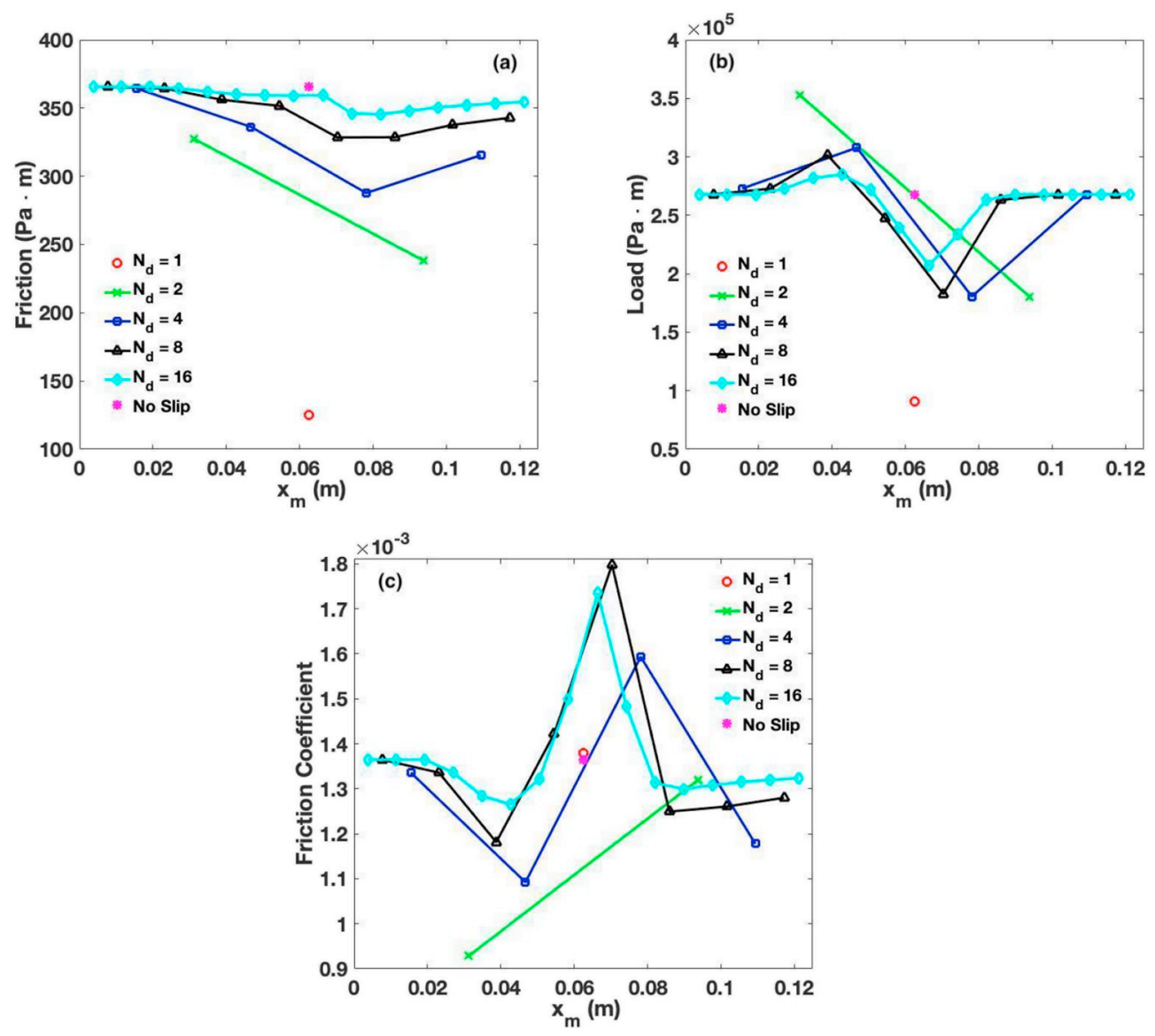

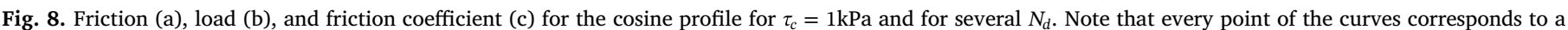

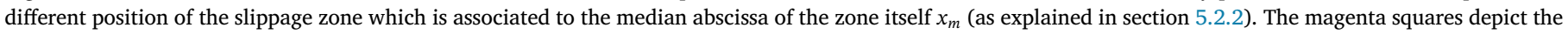
no-slip case.

deteriorates by decreasing the critical shear stress; however, eventually the trend is inverted and for $\tau_{c}=1 \mathrm{kPa}$ we obtain the same value of $F C$ than for $\tau_{c}=\infty$. If $\tau_{c}$ is lowered even more, FC becomes lower than the case with pure no-slip conditions. This shows how slip at the wall can improve the performance of the bearing by decreasing the friction in certain conditions when the load is not minimised.

\subsubsection{Non-uniform critical shear stress}

In the previous section we noted how slippage at the upper wall could be helpful in improving lubrication performance. A slippage condition could be obtained by using superoleophobic surfaces, see for instance Ref. [15]. However this kind of surfaces are expensive and not easy to be manufactured $[39,40]$. For this reason it would be worthwhile to be able to optimise the surface in order to reach good lubrication performance (i.e. low $F C$ ) while minimising the superoleophobic region.

To this goal we illustrate in Fig. 8 the friction (a), the load (b) and the friction coefficient (c) for several $N_{d}$ for $\tau_{c}=1 \mathrm{kPa}$. We have chosen this value of the critical shear stress since it has a similar friction coefficient than the no-slip case. In particular every point depicts a different configuration in which the slippage zone is on a different $1 / N_{d}$-th of the profile. For instance the third point of the black line depicts the case " $N_{d}=8,3 / 8$ ". Note that each configuration is associated to the median abscissa of the slippage zone $x_{m}$. For instance for the case " $N_{d}=8,3 / 8$ " the corresponding abscissa is $x_{m}=\left(\frac{3}{8}-\frac{1}{16}\right) a=3.90625 \cdot 10^{-2} \mathrm{~m}$. The friction is always (i) larger for any partial slip region configuration with respect to $N_{d}=1$ (red circle), while (ii) lower than the no slip case (magenta square). However the load is minimal for $N_{d}=1$. Interestingly the lowest values of $F C$ are for super-oleophobic regions in the second quarter of the bearing. Particularly the case " $N_{d}=2,1 / 2$ ", " $N_{d}=4,2 / 4$ ", " $N_{d}=8,3 / 8$ " and " $N_{d}=16,6 / 16$ " are the configurations for each $N_{d}$ in which $F C$ is minimal. They show a gain in $F C$ with respect to both the no slip and $N_{d}=1$ cases of $31 \%, 20 \%, 13 \%$ and $7 \%$, respectively, but having a significant shorter slippage zone.

Finally we show in Fig. 9 (a) the pressure, (b) the shear stress, (c) the slip velocity and (d) the density ratio for the cases with the lowest $F C$ observed in Fig. 8c: " $N_{d}=2,1 / 2$ ", " $N_{d}=4,2 / 4$ ", " $N_{d}=8,3 / 8$ " and " $N_{d}=16,6 / 16$ ". It is important to notice that in all these cases the slippage zone is in the second quarter of the domain, where cavitation does not occur. The pressure peak increases when slippage is implemented just in one specific region of the domain. This is consistent with the larger load observed in Fig. 8b. In particular this pressure peak - in which the pressure gradient has a discontinuity - corresponds to the end of the slippage zone (see Fig. 9b and c). Eq. (2) is valid in correspondence of the peak with (i) $r=0$, since the peak is in the active region, and (ii) $f(p)=1$ due to the incompressibility of the liquid. Since the value of $h$ does not vary at the two sides of the jump, a negative jump in the slip velocity entails then a negative jump in the pressure gradient. For this reason the pressure has to stop its growth and 

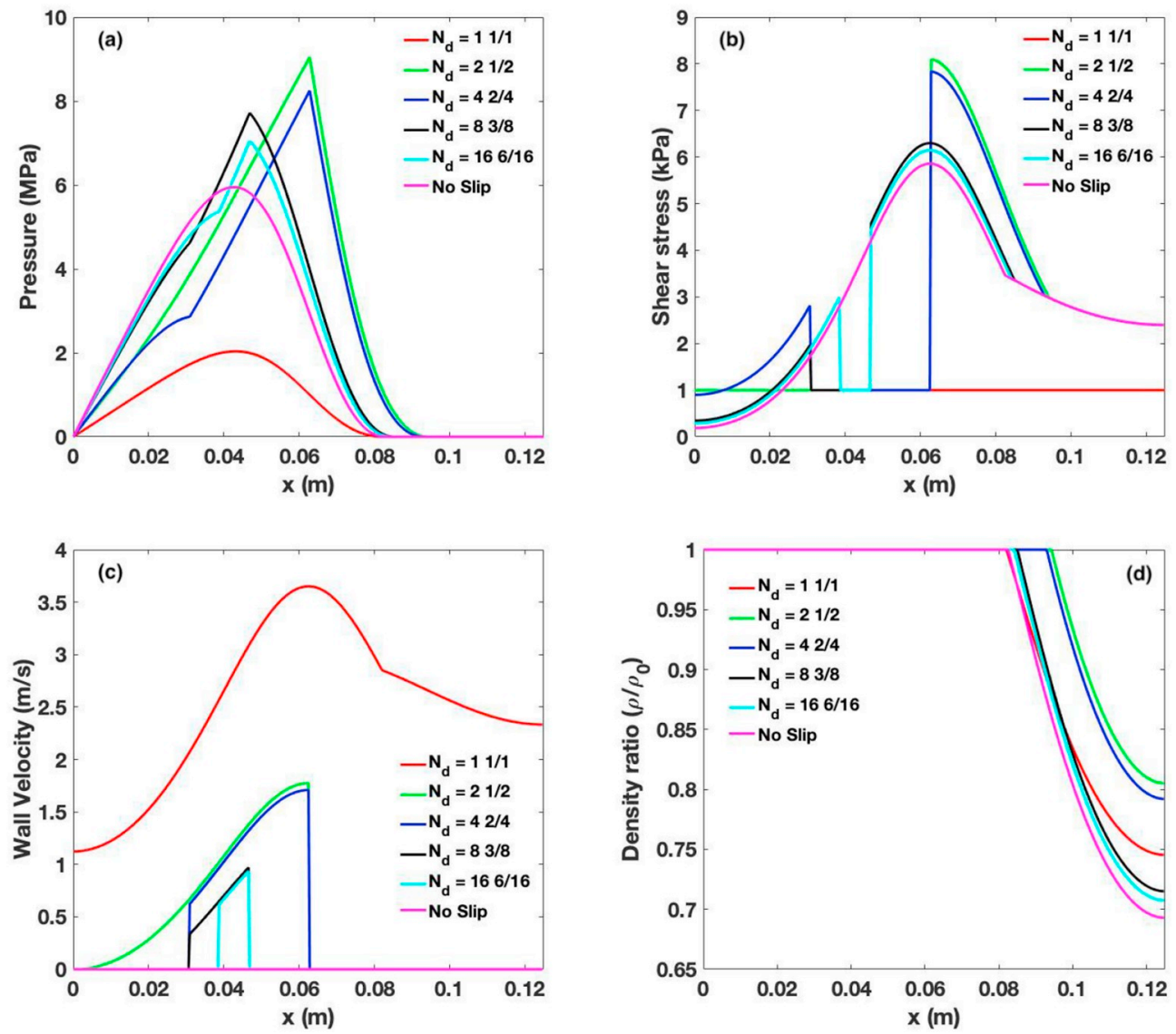

Fig. 9. Pressure (a), shear stress (b), slip velocity (c) and density ratio (d) for the cosine profile for $\tau_{c}=1 \mathrm{kPa}$ and for the cases with the lowest $F C$ observed in Fig. $8 \mathrm{c}$. The magenta line depicts the no slip case.

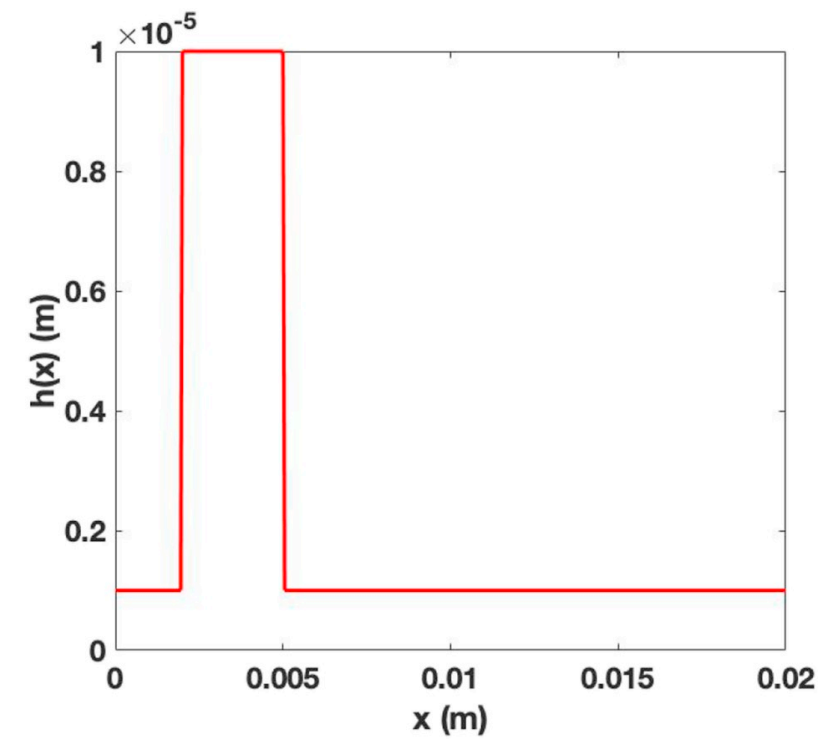

Fig. 10. The pocket profile used to mimic a basic surface textured contact.

decrease. In conclusion a slip/no slip boundary behaves as a step bearing creating a wedge effect [14]. The pressure increase retards also the cavitation appearance (Fig. 9d). Particularly for the cases in which the jump in the slip velocity is larger (green and blue lines) the nonactive region is shifted downstream of about $10 \%$. This also plays a role in improving the lubrication performance observed in Fig. 8.
A discussion on the value of the critical shear stress is however necessary at this point. There is very little literature regarding critical shear stress, and what values are actually achievable [16]. Furthermore, there is no clear information whether $\tau_{c}$ varies with the conditions under which the system operates. However, the manufacturing cost will strongly increase if very small values of $\tau_{c}$ are needed to be reached. Our model shows that "reasonable" values of $\tau_{c}$ (much larger than zero) are needed to achieve a significant decrease of the friction coefficient.

\subsection{Pocketed slider}

In this section we work on the pocketed slider, which is characterised by the profile shown in Fig. 10:

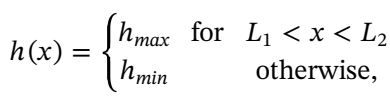

in which $L_{1}=2 \cdot 10^{3} \mathrm{~m}, L_{2}=3 \cdot 10^{3} \mathrm{~m}, h_{\max }=1 \cdot 10^{-} 5 \mathrm{~m}, h_{\min }=1 \cdot 10^{-} 6 \mathrm{~m}$. The length of the domain $a$ was settled to $a=2 \cdot 10^{-4} \mathrm{~m}$, the viscosity $\mu=0.01 \mathrm{~Pa} \cdot \mathrm{s}, U=1 \mathrm{~m} / \mathrm{s}$ and $p_{a}=p_{0}=10^{5} \mathrm{~Pa}$. This profile was already used by Giacopini et al. [35] with setting no slip conditions at both boundaries. In section 5.3.1 we report the results with considering a uniform critical shear stress, while in section 5.3.2 the critical shear stress varies along the $x$-direction.

\subsubsection{Uniform critical shear stress}

In Fig. 11 we report the pressure (a), the shear stress (b), the slip velocity (c) and the density ratio (d) for several values of the critical 

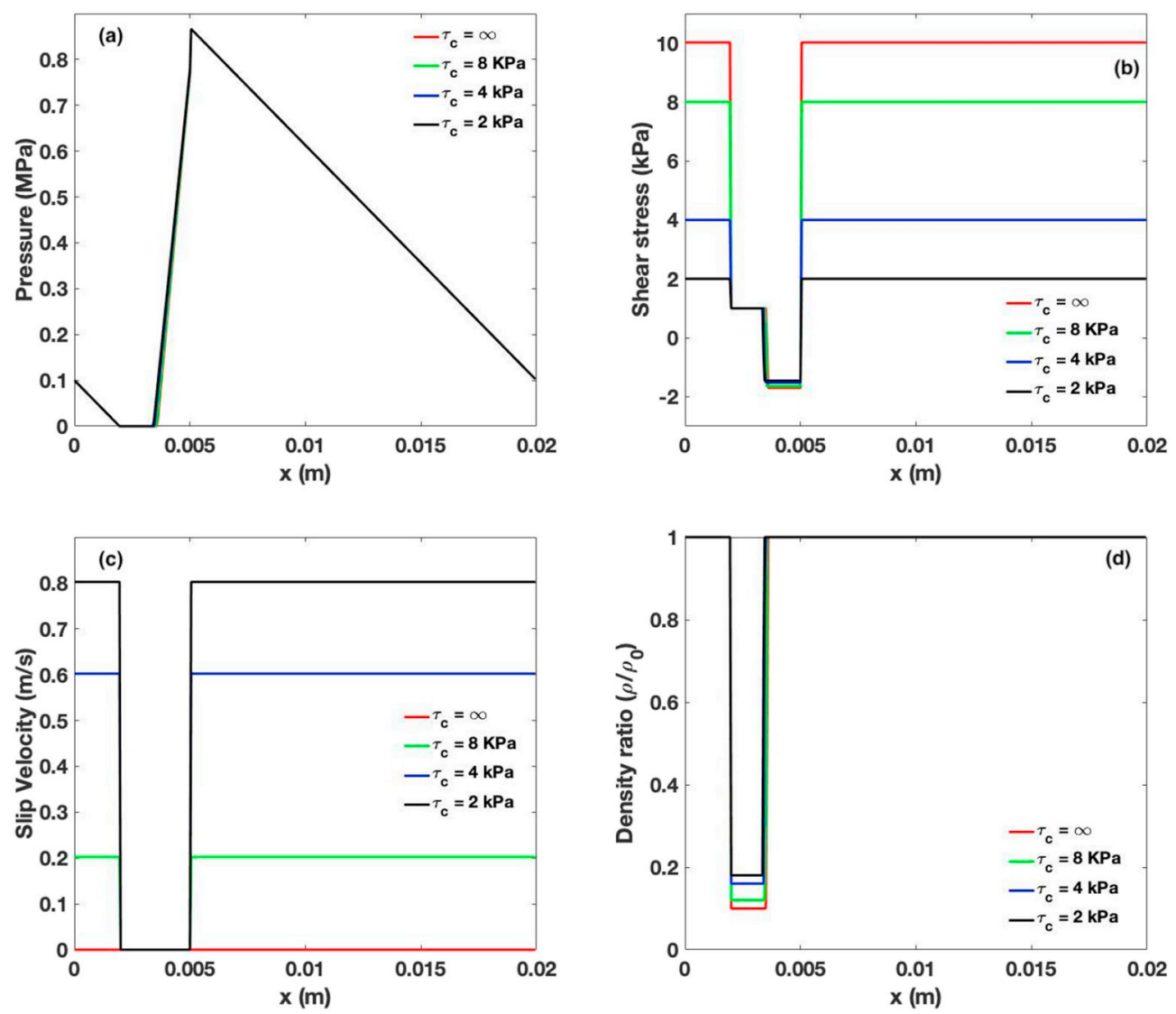

Fig. 11. Pressure (a), shear stress (b), slip velocity (c) and density ratio (d) for the pocket profile for several values of uniform $\tau_{c}$.
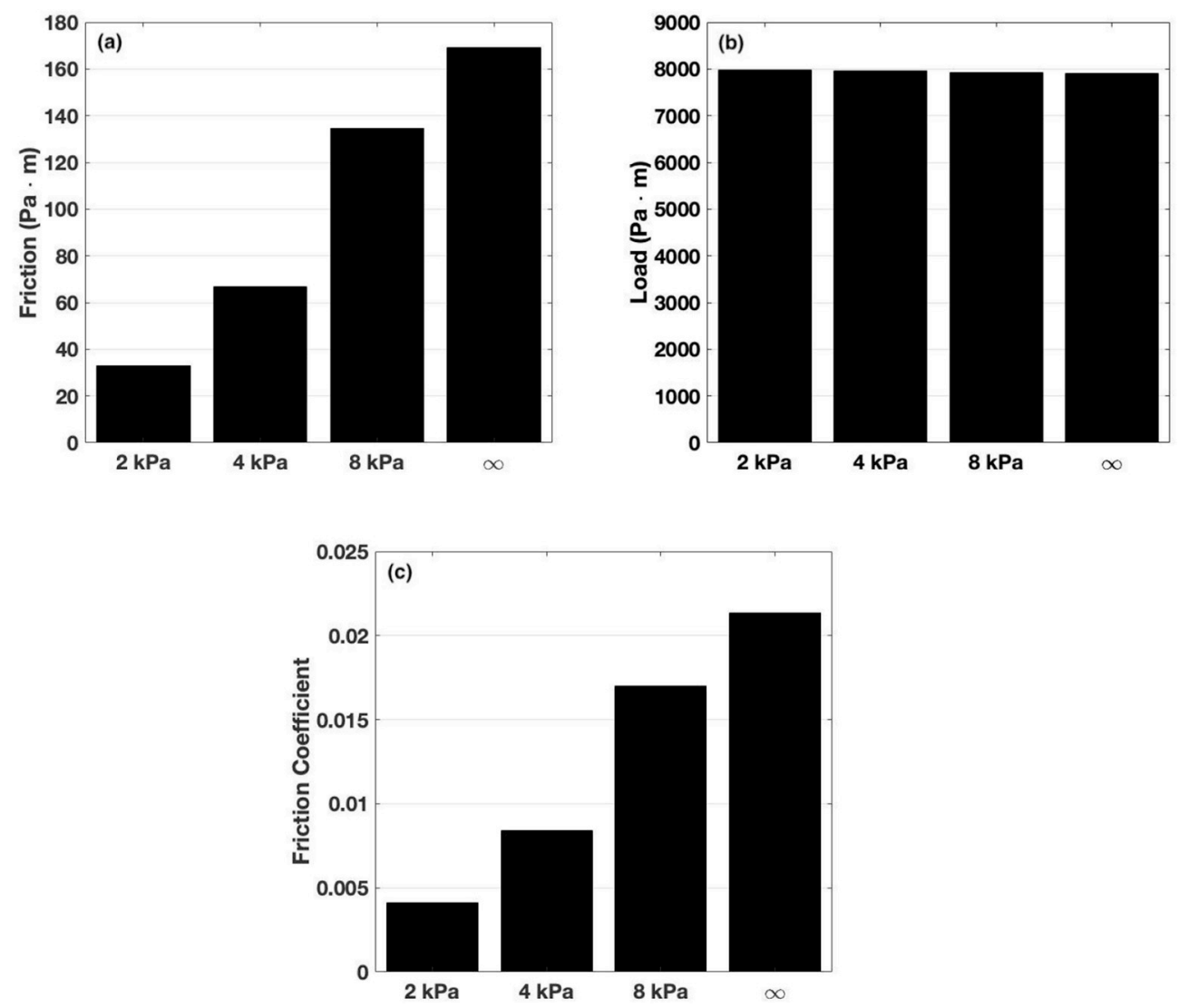

Fig. 12. Friction (a), load (b), and friction coefficient (c) for the pocket profile for several values of uniform $\tau_{c}$. 

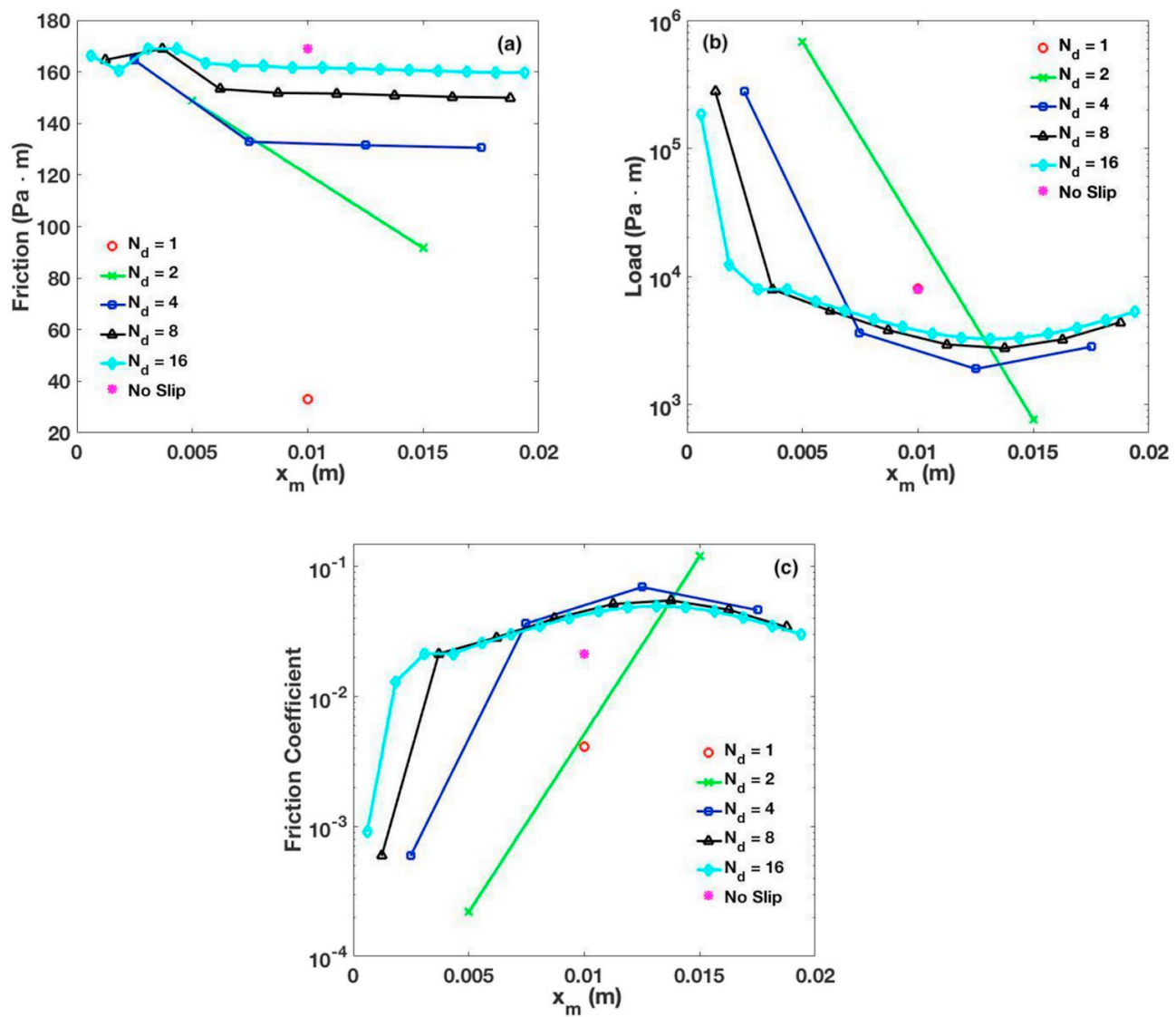

Fig. 13. Friction (a), load (b), and friction coefficient (c) for the pocket profile for $\tau_{c}=2 \mathrm{kPa}$ and for several values of $N_{d}$. Note that every point of the curves corresponds to a different position of the slippage zone which is associated to the median abscissa of the zone itself $x_{m}$ (as explained in section 5.2.2). The magenta squares depict the no slip case.

shear stress (assumed constant). The case with $\tau_{c}=\infty$ has been validated by comparisons with the analytical solution [41] and the simulations of Ref. [35]. The pressure and the size of the non-active region does marginally depend on the presence of a slippage zone. The shear stress instead and, as a consequence, the slip velocity are strongly effected by $\tau_{c}$.

The friction (a), the load (b) and the friction coefficient (c) are shown in Fig. 12 for the same values of $\tau_{c}$ of Fig. 11. Not surprisingly the effect of the slippage region on the friction (load) is in agreement to its influence on the shear stress (pressure), see Fig. 11b (11a). Since the friction is always decreasing with lowering $\tau_{c}$, while the load is in practice constant, then a lower $\tau_{c}$ is beneficial to improve the friction coefficient values.

\subsubsection{Non-uniform critical shear stress}

In this section non-uniform values of the critical shear stress $\tau_{c}(x)$ were assumed. In particular $\tau_{c}$ has finite value only in a region of the domain similarly to section 5.2 .2 for the cosine profile.

Fig. 13 shows the value of the friction (a), the load (b) and the friction coefficient (c) for several values of $N_{d}$ and for $\tau_{c}=2 \mathrm{kPa}$, compared with the pure no slip case (magenta squares). The friction is minimised by the case $N_{d}=1$, i.e. where the slippage could occur in all the upper wall, and more in general low values of $F$ are obtained when the slippage occurs towards the end of the domain. However the load is the parameter more affected by both $N_{d}$ and the position of the slippage zone. If the slippage zone is located close to the entry of the domain the load could increase by one or two orders of magnitudes with respect to the uniform critical shear stress case depending on the number of parts in which the domain is divided. The much higher values of the load correspond to significantly lower friction coefficient values, in particular one/two orders of magnitude lower than $N_{d}=1$. On the other hand if the slippage zone is located close to the exit the performance of the contact strongly diminishes due to the lost load capacity.

To understand the reason of this behaviour we trace in Fig. 14 the pressure (a), the shear stress (b), the slip velocity (c) and the density ratio (d) for the cases with the lowest $F C$ of Fig. 13: " $N_{d}=1,1 / 1$ ", " $N_{d}=2,1 / 2$ ", " $N_{d}=4,1 / 4$ ", " $N_{d}=8,1 / 8$ " and " $N_{d}=16,1 / 16$ ". In all these cases the slippage region is located close to the entry. The pressure peak strongly increases by imposing the slippage only in a specific location of the domain and its location is determined by the end of the slippage zone. This behaviour is due to the negative jump in $U_{s}$ as explained in section 5.2.2. Interestingly cavitation does not occur when $N_{d}>1$ since the pressure is too high. This is another reason of the large increase in load capacity noticed in Fig. 13b.

Finally in Fig. 15 the pressure (a), the shear stress (b), the slip velocity (c) and the density ratio (d) for the configurations in which the slippage zone is closer to the exit: " $N_{d}=1,1 / 1$ ", " $N_{d}=2,2 / 2$ ", " $N_{d}=4$, $4 / 4$ ", " $N_{d}=8,8 / 8$ " and " $N_{d}=16,16 / 16$ ". The pressure decreases if the slippage zone is only at the end of the domain. This explains the minor load capacity observed in Fig. 11b. Moreover cavitation does occur also in the slippage region and not just in the pocket as for $N_{d}=1$ and for the no slip cases. The onset of the second non-active region corresponds to the positive jump in slip velocity (see Fig. 15c). This is in agreement with the findings of Bayada \& Meurisse [14]: a slip zone located 

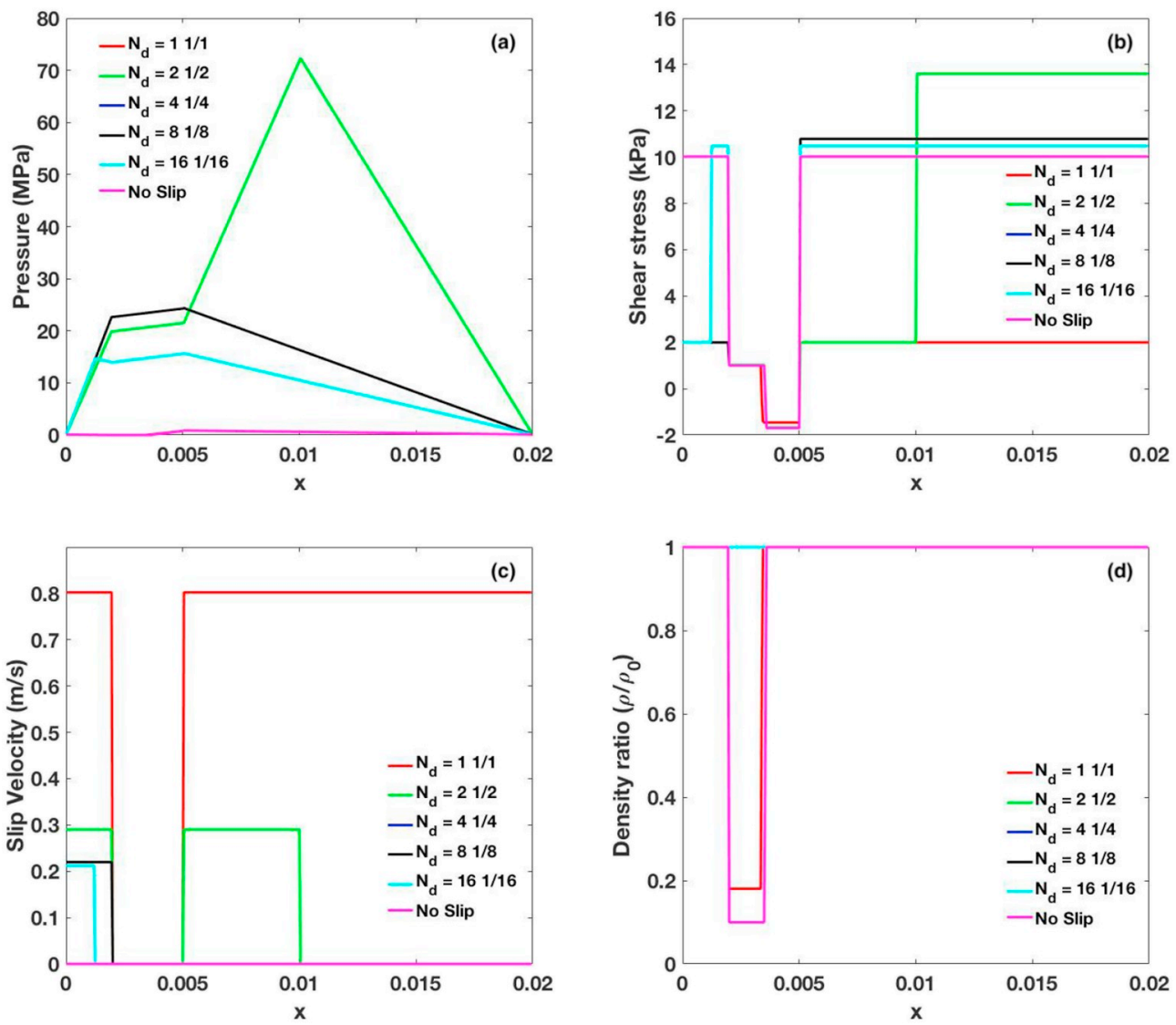

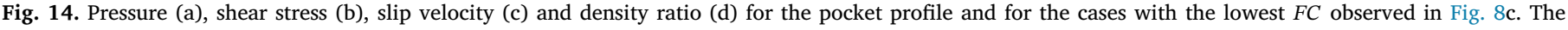

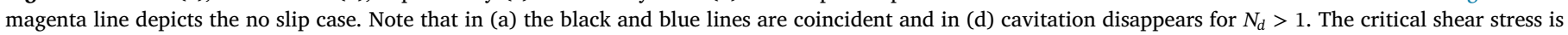
$\tau_{c}=2 \mathrm{kPa}$. (For interpretation of the references to colour in this figure legend, the reader is referred to the Web version of this article.)

downstream from a no-slip zone tends to reduce the pressure like diverging geometry does, and this can lead to the occurrence of cavitation. Thus it should be strongly avoided to locate the slippage in proximity of the contact exit.

\subsection{Multi-pocketed slider}

In this section we illustrate results obtained for a multi-pocket profile similar to one of the cases studied by Tauviqirrahman et al. [25]. The multi-pocket profile is described by

$h(x)=\left\{\begin{array}{lc}h_{\max } \text { for } & b<x<2 b \cup 3 b<x<4 b \cup 5 b<x<6 b \\ h_{\min } & \text { otherwise }\end{array}\right.$

and it is shown schematically in Fig. 16. Studying this particular profile we can (i) approach a more realistic textured surfaces with respect to the single pocket studied in section 5.3and (ii) show the predictive capabilities of our cavitation algorithm, since this kind of profile can only be adequately described using mass-conserving cavitation algorithms.

In Fig. 17 we illustrate the (a) pressure, (b) shear stress, (c) slip velocity and (d) density ratio for the multi-pocket profile for different values of uniform $\tau_{c}$. The pressure profile (shear stress) increases (decreases) by lowering the value of the critical shear stress. The qualitative effect of increasing the slippage (i.e. decreasing $\tau_{c}$ ) on the slip velocity and the density ratio is similar to what observed for the single pocket case (see Fig. 11c and d).
Finally in Fig. 18 the (a) friction, (b) load and (c) friction coefficient are showed for the same values of $\tau_{c}$ used in Fig. 17. While the friction shows the same trends obtained for the single pocket profile, i.e. it reduces as $\tau_{c}$ is lowered (see Fig. 11a), the load behaves differently than the single pocket when slippage is introduced, since a significant load increase is observed. Both these factors result in a strong decrease of the friction coefficient for superoleophobic multi-pocketed surfaces. Particularly the friction coefficient for $\tau_{c}=0.5 \mathrm{kPa}$ is less than $25 \%$ of the case characterised by no $\operatorname{slip}\left(\tau_{c}=\infty\right)$.

\section{Conclusions}

In this work a model capable to predict the behaviour of bearings in which both cavitation and slip at the wall occur has been proposed. The main novelty of our approach is the complementary formulations used to describe these phenomena. In particular, to model the possible slippage we have used the Tresca model and a critical shear stress value $\tau_{c}$ above which the fluid slips at the upper wall. We summarise below our findings.

- The algorithm was validated by comparison with Bayada [31] for a twin parabolic slider;

- We have first conducted our analyses by fixing a uniform value of the critical shear stress. We have observed that the lubrication performance for a bearing (modelled as a cosine profile in its hydrodynamic equivalence) and pocketed profiles can improve depending on the value of $\tau_{c}$. In particular, while friction is always 

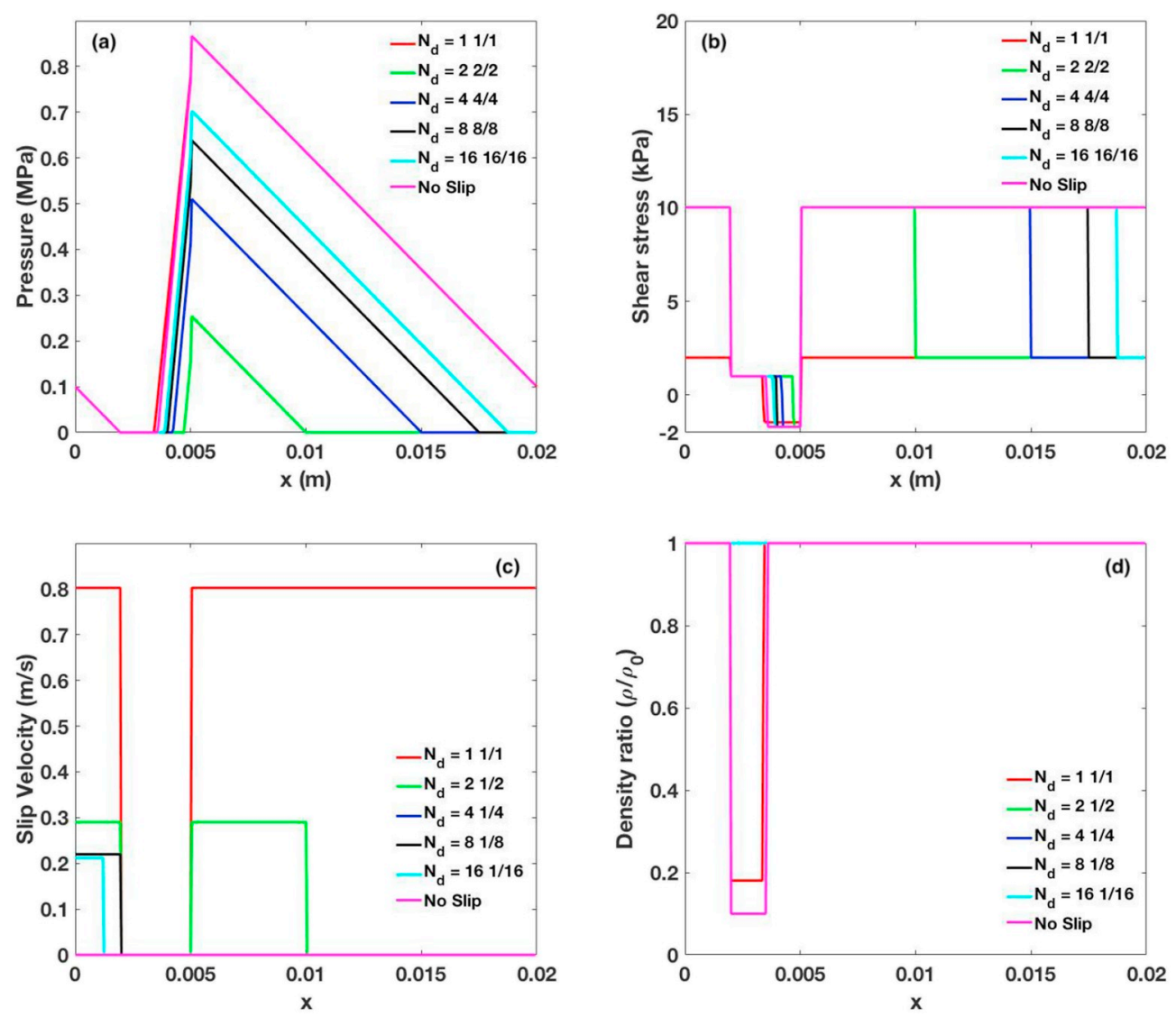

Fig. 15. Pressure (a), shear stress (b), slip velocity (c) and density ratio (d) for the cosine profile when the slippage region is at the end of domain. The magenta line depicts the no slip case. The critical shear stress is $\tau_{c}=2 \mathrm{kPa}$.

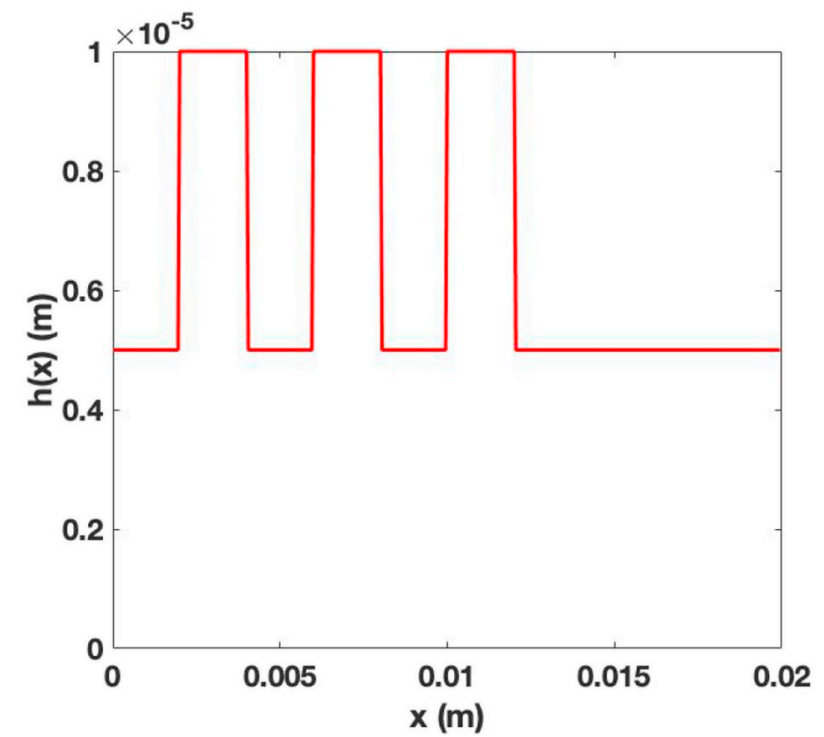

Fig. 16. The multipocket profile used to mimic a basic surface textured contact.

decreasing, load carrying capacity needs to be optimised by choosing an appropriate value of the critical shear stress (therefore, by changing the local surface properties);

- For pocketed sliders the friction is always decreasing if the critical shear stress is lowered. Consequently, the friction coefficient decreases as well since the load is either nearly unchanged or increased by introducing slippage;

- In the subsequent investigations the critical shear stress was assumed to be piecewise constant. We have found that there is an optimal configuration in which the friction coefficient significantly lowers compared to the no-slip case for all configurations. The improved performance depends on a pressure increase (and the consequent load increase). This increase is due to the negative jump in the slip velocity at the end of the slippage zone;

- In the particular case of a multi-poked profile, while the qualitative effects of introducing slippage is similar to the case of a single pocket for shear stress, velocity and density ratio profiles, a very large increase of the pressure profile is registered, which produces a significant decrease of the friction coefficient in the configuration investigated here;

- With these results we have shown that the performance of bearings in which there is interplay between slip at the wall and cavitation can be significantly improved (up to two orders of magnitude reduction of friction coefficient) while minimising the slippage region. This can reduce cost in manufacturing superoleophobic bearings since surface treatments can be confined to specific (small) regions to strongly improve bearings' performance;

- Furthermore, our model has showed that the values of the critical shear stress necessary to achieve a significant decrease of the friction coefficient are not prohibitively small and can be achieved using conventional processes, pointing out further the possibility of reduction in manufacturing cost;

In this paper we have shown the flexibility of our model which can be used also for different contact problems, such as ridges [42] or 

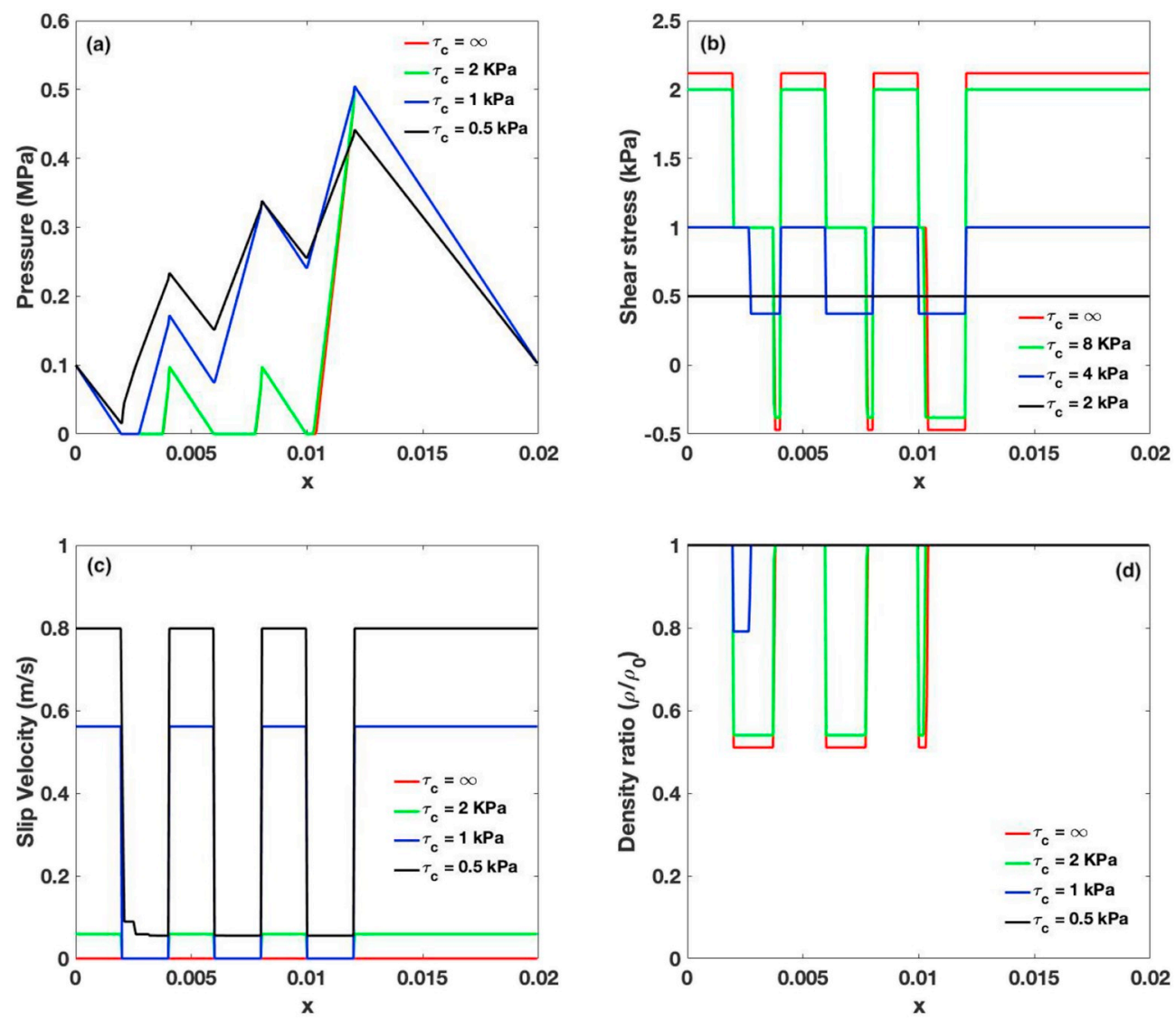

Fig. 17. Pressure (a), shear stress (b), slip velocity (c) and density ratio (d) for the multipocket profile for several values of uniform $\tau_{c}$.
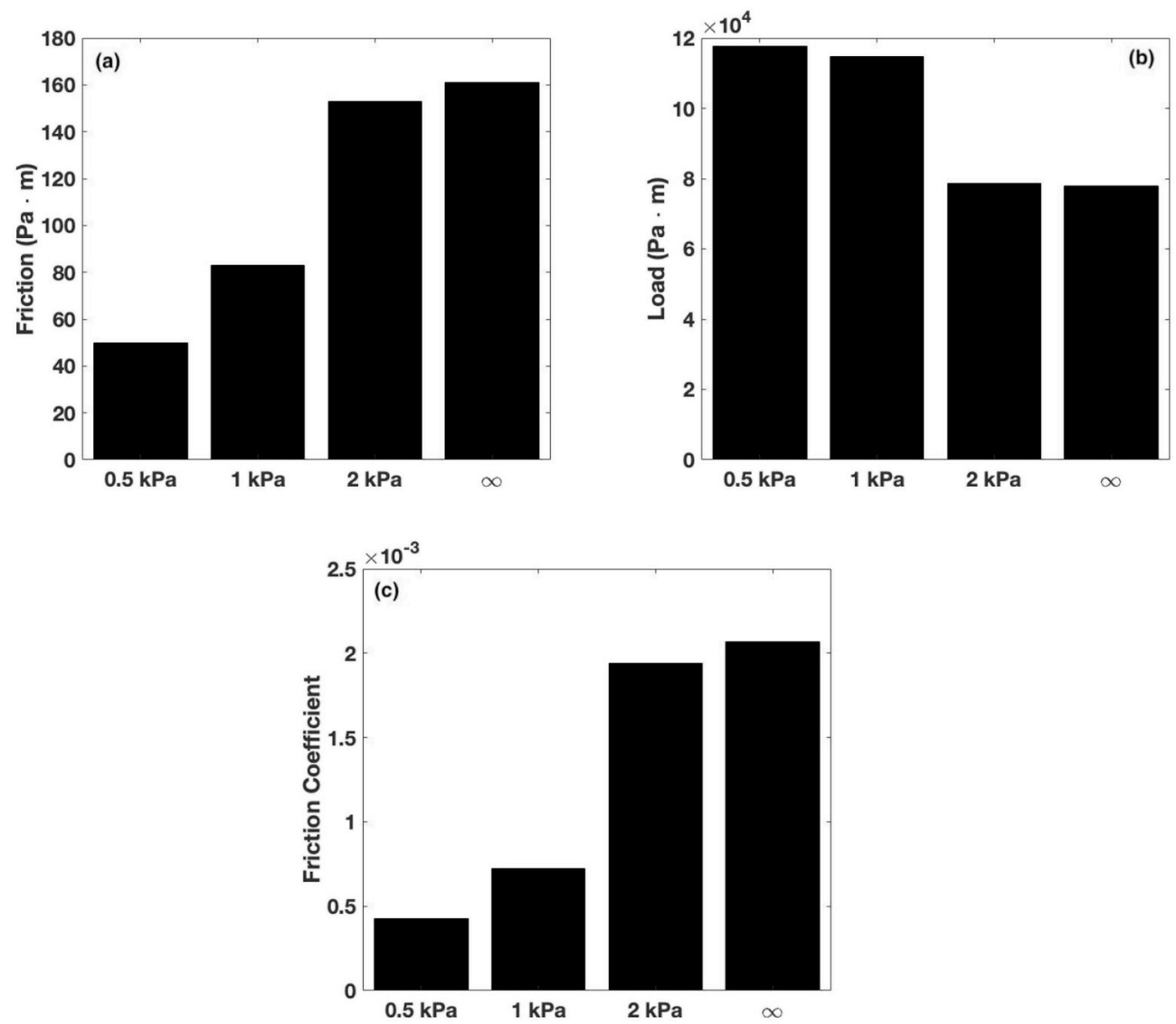

Fig. 18. Friction (a), load (b), and friction coefficient (c) for the multipocket profile for several values of uniform $\tau_{c}$. 
squeezing contacts [36]. Future efforts will be made to couple the present algorithm with a suitable complementary formulation to describe solid elastic deformation and direct asperity contact [32]. and to extend our analyses to finite length bearings. While our mass-conserving cavitation algorithm has already been demonstrated to be able to simulate these cases, some modifications need to be implemented to predict the slippage in two dimensions, see Ref. [20]for more details.

\section{Acknowledgments}

This presented work was supported by the Engineering and Physical Sciences Research Council (EPSRC) through Prof. Dini's Established Career Fellowship [EP/N025954/1]. Data from the article will be made freely available by contacting the corresponding author or by emailing tribology@imperial.ac.uk.

\section{Appendix A. Supplementary data}

Supplementary data to this article can be found online at https://doi.org/10.1016/j.triboint.2019.04.040.

\section{Appendix B. Analytical model for tangential slip}

In this appendix, the modified Reynolds equation which takes into account a possible slippage between the fluid and one of the solid surfaces is derived. Our findings are the same of Ref. [15] and they are here reported for the sake of clarity.

First of all, consider a small element of fluid from a hydrodynamic film located between two moving, solid surfaces:

$\frac{\partial \tau_{x z}}{\partial z}=-\frac{\partial p}{\partial z}$

$\frac{\partial \tau_{y z}}{\partial z}=-\frac{\partial p}{\partial y}$

where $\tau$ is the shear stress and $p$ is the pressure. Refer to Fig. 1 for the convention on the shear stress sign. In this paper the upper surface is stationary and the lower one moves with velocity U. Moreover, the particular case of a possible slippage only between the fluid and the upper fixed surface is considered. Since the Tresca model is employed in order to mimic this slippage, a particular critical shear stress along both the $x$-direction, $\tau_{c x}$, and the $y$-direction, $\tau_{c y}$, has to be defined. Note that when perfect slippage is considered, $\tau_{c x}=\tau_{c y}=0$.

By integrating Equation (B.1a) and applying the boundary condition $\tau_{x z}=\tau_{c x}$ at $z=h$ one obtains

$\tau_{x z}=-\frac{\partial p}{\partial x}(z-h)+\tau_{c x}$.

We consider the expression for the viscous shear stress, $\tau_{x z}=-\mu \frac{\partial u}{\partial z}$, where $\mu$ is the dynamic shear viscosity and $u$ is the fluid velocity. Equation (B.2) can be thus rearranged as:

$-\mu \frac{\partial u}{\partial z}=-\frac{\partial p}{\partial x}(z-h)+\tau_{c x}$.

The viscosity is assumed to be constant along the $z$-direction and the velocity at the wetted, no-slip, moving, lower surface, i.e. $z=0$, is $u=U$. The integration of Equation (B.3) gives:

$u=\frac{1}{\mu} \frac{\partial p}{\partial x}\left(\frac{z^{2}}{2}-h z\right)-\frac{\tau_{c x}}{\mu} z+U$

A similar procedure can be followed for the $y$-direction.

By substituting $z=h$ in Equation (B.4), the velocity of the fluid layer in contact with the upper slipping surface in the $x$-direction, $U_{s}$, can be obtained:

$U_{s}=-\frac{h^{2}}{2 \mu} \frac{\partial p}{\partial x}-\tau_{c x} \mu h+U$

Equation (B.4) can than be integrated across the film thickness to obtain the flow in the $x$-direction through a single column of fluid:

$q_{x}=-\frac{h^{3}}{3 \mu} \frac{\partial p}{\partial x}-\frac{\tau_{c x} h^{2}}{2 \mu}+U h$

By differentiating Equation (B.6) one obtains:

$\frac{\partial q_{x}}{\partial x}=-\frac{\partial}{\partial x}\left(\frac{h^{3}}{3 \mu} \frac{\partial p}{\partial x}\right)-\frac{\partial}{\partial x}\left(\frac{\tau_{c x} h^{2}}{2 \mu}\right)+\frac{\partial(U h)}{\partial x}$.

An equivalent expression can be obtained for the $y$-direction.

Finally by applying the continuity of flow principle and supposing that no lubricant is supplied in the $z$-direction:

$\frac{\partial q_{x}}{\partial x}+\frac{\partial q_{y}}{\partial y}=0$ 
Substituting Equation (B.7) and its analogue in the $y$-direction into Equation (B.8) the modified Reynold equation for the slippage problem is derived:

$$
\begin{gathered}
\frac{\partial}{\partial x}\left(\frac{h^{3}}{3 \mu} \frac{\partial p}{\partial x}\right)+\frac{\partial}{\partial y}\left(\frac{h^{3}}{3 \mu} \frac{\partial p}{\partial y}\right)=\frac{\partial(U h)}{\partial x}+\frac{\partial(V h)}{\partial y}+ \\
-\frac{\partial}{\partial x}\left(\frac{\tau_{c x} h^{2}}{2 \mu}\right)-\frac{\partial}{\partial y}\left(\frac{\tau_{c y} h^{2}}{2 \mu}\right) .
\end{gathered}
$$

Equation (B.9) can be then simplified considering the infinitely long bearing approximation, i.e. $\frac{\partial p}{\partial y}=\frac{\partial h}{\partial y}=0$, and assuming a constant viscosity over the bearing area (i.e. $\mu$ is independent of $x$ )

$\frac{d}{d x}\left(h^{3} \frac{d p}{d x}\right)=3 U \mu \frac{\partial h}{\partial x}-\frac{3 \tau_{c}}{2} \frac{d\left(h^{2}\right)}{d x}$.

\section{References}

[1] Holmberg K, Erdemir A. Influence of tribology on global energy consumption, costs and emissions. Friction 2017;5(3):263-84.

[2] Holmberg K, Andersson P, Erdemir A. Global energy consumption due to friction in passenger cars. Tribol Int 2012;47:221-34.

[3] Vakis AI, Yastrebov VA, Scheibert J, Nicola L, Dini D, Minfray C, Almqvist A, Paggi M, Lee SW, Limbert G, et al. Modeling and simulation in tribology across scales: an overview. Tribol Int 2018;125:169-99.

[4] Ewen JP, Heyes DM, Dini D. Advances in nonequilibrium molecular dynamics simulations of lubricants and additives. Friction 2018:1-38.

[5] Wang W, Wang Y, Bao H, Xiong B, Bao M. Friction and wear properties in mems. Sensor Actuator Phys 2002;97:486-91.

[6] Borghi A, Gultieri E, Marchetto D, Moretti L, Valeri S. Tribological effects of surface texturing on nitriding steel for high-performance engine applications. Wear 2008;265(7-8):1046-51.

[7] Ou J, Rothstein JP. Direct velocity measurements of the flow past drag-reducing ultrahydrophobic surfaces. Phys Fluids 2005;17(10):103606.

[8] Etsion I. State of the art in laser surface texturing. J Tribol 2005;127(1):248-53.

[9] Etsion I. Modeling of surface texturing in hydrodynamic lubrication. Friction 2013;1(3):195-209.

[10] Vladescu S-C, Olver AV, Pegg IG, Reddyhoff T. The effects of surface texture in reciprocating contacts-an experimental study. Tribol Int 2015;82:28-42.

[11] Medina S, Fowell M, Vladescu S-C, Reddyhoff T, Pegg I, Olver AV, Dini D. Transient effects in lubricated textured bearings. Proc IME J J Eng Tribol 2015;229(4):523-37.

[12] Vladescu S-C, Ciniero A, Tufail K, Gangopadhyay A, Reddyhoff T. Optimization of pocket geometry for friction reduction in piston-liner contacts. Tribol Trans 2018;61(3):522-31.

[13] Rothstein JP. Slip on superhydrophobic surfaces. Annu Rev Fluid Mech 2010;42:89-109.

[14] Bayada G, Meurisse M-H. Impact of the cavitation model on the theoretical performance of heterogeneous slip/no-slip engineered contacts in hydrodynamic conditions. Proc IME J J Eng Tribol 2009;223(3):371-81.

[15] Spikes HA. The half-wetted bearing. part 1: extended Reynolds equation. Proc IME J J Eng Tribol 2003;217(1):1-14.

[16] Spikes HA. The half-wetted bearing. part 2: potential application in low load contacts. Proc IME J J Eng Tribol 2003;217(1):15-26.

[17] Li W-L, Chu H-M, Chen M-D. The partially wetted bearingextended Reynolds equation. Tribol Int 2006;39(11):1428-35.

[18] Jao H-, Chang K-M, Chu L-M, Li W-L. A modified average Reynolds equation for rough bearings with anisotropic slip. J Tribol 2016;138(1):011702.

[19] Ma GJ, Wu CW, Zhou P. Wall slip and hydrodynamics of two-dimensional journal bearing. Tribol Int 2007;40(7):1056-66.

[20] Ma GJ, Wu CW, Zhou P. Multi-linearity algorithm for wall slip in two-dimensional gap flow. Int J Numer Methods Eng 2007;69(12):2469-84.

[21] Savio D, Falk K, Moseler M. Slipping domains in water-lubricated microsystems for improved load support. Tribol Int 2018;120:269-79.

[22] Rao TVVLN. Analysis of single-grooved slider and journal bearing with partial slip surface. J Tribol 2010;132(1):014501.
[23] Senatore A, Rao TVVLN. Partial slip texture slider and journal bearing lubricated with Newtonian fluids: a review. J Tribol 2018;140(4):040801.

[24] Aurelian F, Patrick M, Mohamed H. Wall slip effects in (elasto) hydrodynamic journal bearings. Tribol Int 2011;44(7-8):868-77.

[25] Tauviqirrahman M, Ismail R, Jamari J, Schipper DJ. Combined effect of texturing and boundary slippage in lubricated sliding contacts. Tribol Int 2013;66:274-81.

[26] Tauviqirrahman M, Ismail R, Jamari J, Schipper DJ. A study of surface texturing and boundary slip on improving the load support of lubricated parallel sliding contacts. Acta Mech 2013;224(2):365-81.

[27] Susilowati S, Tauviqirrahman M, Jamari J, Bayuseno AP. Numerical investigation of the combined effects of slip and texture on tribological performance of bearing. Tribol Mater Surface Interfac 2016;10(2):86-9.

[28] Muchammad M, Tauviqirrahman M, Jamari J, Schipper DJ. An analytical approach on the tribological behaviour of pocketed slider bearings with boundary slip including cavitation. Lubric Sci 2017;29(3):133-52.

[29] Muchammad M, Tauviqirrahman M, Jamari J, Schipper DJ. Numerical investigation of texturing and wall slip in lubricated sliding contact considering cavitation. Tribol Online 2017;12(2):67-75.

[30] Lin Q, Wei Z, Wang N, Chen W. Effect of large-area texture/slip surface on journal bearing considering cavitation. Ind Lubr Tribol 2015;67(3):216-26.

[31] Bayada G. A fast algorithm for boundary slippage including mass flow conserving cavitation model. Tribol Int 2018;118:71-88.

[32] Cannarozzi AA. On the resolution of some unilaterally constrained problems in structural engineering. Comput Methods Appl Mech Eng 1980;24(3):339-57.

[33] Strozzi A. Formulation of three lubrication problems in terms of complementarity. Wear 1985;104(2):103-19.

[34] Strozzi A, Giacopini M, Bertocchi E, Dini D. Formulation of the tangential velocity slip problem in terms of variational inequalities. Proc IME J J Eng Tribol 2014;228(10):1122-35.

[35] Giacopini M, Fowell MT, Dini D, Strozzi A. A mass-conserving complementarity formulation to study lubricant films in the presence of cavitation. J Tribol 2010;132(4):041702.

[36] Bertocchi L, Dini D, Giacopini M, Fowell MT, Baldini A. Fluid film lubrication in the presence of cavitation: a mass-conserving two-dimensional formulation for compressible, piezoviscous and non-Newtonian fluids. Tribol Int 2013;67:61-71.

[37] Oden JT, Kikuchi N. Theory of variational inequalities with applications to problems of flow through porous media. Int J Eng Sci 1980;18(10):1173-284.

[38] Bayada G, Chupin L. Compressible fluid model for hydrodynamic lubrication cavitation. J Tribol 2013;135(4):041702.

[39] Wang S, Feng L, Jiang L. One-step solution-immersion process for the fabrication of stable bionic superhydrophobic surfaces. Adv Mater 2006;18(6):767-70.

[40] Qu M, Zhang B, Song L, Chen S, Zhang J, Cao X. Fabrication of superhydrophobic surfaces on engineering materials by a solution-immersion process. Adv Funct Mater 2007;17(4):593-6.

[41] Olver AV, Fowell MT, Spikes HA, Pegg IG. 'inlet suction', a load support mechanism in non-convergent, pocketed, hydrodynamic bearings. Proc IME J J Eng Tribol 2006;220(2):105-8.

[42] Almqvist T, Larsson R. Some remarks on the validity of Reynolds equation in the modeling of lubricant film flows on the surface roughness scale. J Tribol 2004;126(4):703-10. 